\title{
Fractional Model and Exact Solutions of Convection Flow of an Incompressible Viscous Fluid under the Newtonian Heating and Mass Diffusion
}

\author{
Ndolane Sene (iD \\ Department of Mathematics, Cheikh Anta Diop University, BP 5683 Dakar Fann, Senegal \\ Correspondence should be addressed to Ndolane Sene; ndolanesene@yahoo.fr
}

Received 29 October 2021; Revised 19 November 2021; Accepted 29 November 2021; Published 12 January 2022

Academic Editor: Saima Arshed

Copyright (c) 2022 Ndolane Sene. This is an open access article distributed under the Creative Commons Attribution License, which permits unrestricted use, distribution, and reproduction in any medium, provided the original work is properly cited.

\begin{abstract}
In this paper, we consider a natural convection flow of an incompressible viscous fluid subject to Newtonian heating and constant mass diffusion. The proposed model has been described by the Caputo fractional operator. The used derivative is compatible with physical initial and boundaries conditions. The exact analytical solutions of the proposed model have been provided using the Laplace transform method. The obtained solutions are expressed using some special functions as the Gaussian error function, Mittag-Leffler function, Wright function, and $G$-function. The influences of the order of the fractional operator, parameters used in modeling the considered fluid, Nusselt number, and Sherwood number have been analyzed and discussed. The physical interpretations of the influences of the parameters of our fluid model have been presented and analyzed as well. We use the graphical representations of the exact solutions of the model to support the findings of the paper.
\end{abstract}

\section{Introduction}

The main challenge during these decades is modeling realworld phenomena using fractional operators. This challenge is motivated by the memory effect observed in fractional operators and the generalization of the differential equations to noninteger order derivatives. Many explanations and tentatives to explain the utilities of fractional calculus have been addressed in the literature. The readers are advised to take a look at the following application papers in biology $[1,2]$, science and engineering [3, 4], physics [5-7], mathematical physics [8-10], and many others domains $[11,12]$. Fractional calculus is also a field with many controversies and discussions on the validity of fractional operators. Note that there exist many fractional operators in the literature: fractional operators with singularities as the Riemann-Liouville derivative and the Caputo fractional derivative $[13,14]$. There also exist many generalizations of the previous operators; see [15]. Fractional operators without singularities are known as the fractional operator with exponential kernel proposed by Caputo-Fabrizio and the fractional operator with Mittag-Leffler kernel provided by Atangana and Baleanu [16-18]. All cited operators have their advantages and their conveniences reported in the literature $[16,19]$. The present paper focuses on applying the Caputo derivative to fluids models. The first reason for using this fractional operator is the memory effect, and the second reason is that this operator is more realistic because the derivative of a constant is zero, contrary to the Riemann-Liouville operator in which the derivative of a constant is not zero.

In the literature, there exist many papers in the same directions. We recall some of them, which will permit us to see the findings addressed in the literature and the advantages of our present paper regarding the existing literature. Khan et al. [19] model heat and mass transfer of secondgrade fluids over a vertical plate using derivative with exponential kernel and derivative with Mittag-Leffler kernel. They have used the Laplace transforms to get the analytical solutions of the proposed model. Samiulhaq et al. [20] studied the magnetic field influence on unsteady free convection flow of a second-grade fluid near an infinite vertical 
flat plate with ramped wall temperature embedded in a porous medium. The Laplace transform method has been used in the investigations related to the exact solutions of the model considered in the paper. Shah et al. [21] proposed an investigation related to the influence of magnetic field on double convection problem of viscous fluid described by fractional-order derivative over an exponentially moving vertical plate. Zafar et al. [22] proposed the exact solutions of the model described by fractional operator obeying the rotational flow of some fractional Maxwell fluid. Imran et al. [3] proposed the study on the semianalytic solutions of a viscous fluid with old and new definitions of fractional derivatives. Hussanan et al. [23] investigated the soret effects on unsteady magnetohydrodynamic mixed convection heatand-mass-transfer flow in a porous medium with Newtonian heating. Reyaz et al. [24] studied modeling with Caputo operator the MHD Casson fluid flow over an oscillating plate with thermal radiation. Narahari [25] presented the effects of thermal radiation and mass diffusion on free convection flow near a vertical plate with Newtonian heating. Sheikh et al. [26] proposed a comparative study between the fractionalorder derivatives without singularities to the convective flow of a generalized Casson fluid. Ali et al. [9] applied the fractional operation with the exponential kernel to MHD free convection flow of generalized Walters' B fluid model. The authors proposed a fractional model and tried to get solutions using the applications of the Laplace transforms. Abro [27] proposed using fractional operator investigation in analytic investigation of thermodiffusion process on free convection flow. Shen et al. [28] used Fourier sine transform to propose the analytical solutions of the Rayleigh-Stokes problem for a heated generalized second-grade fluid described by the fractional derivative. Vieru et al. [29] first proposed an investigation related to the analytical solutions of the fractional model described free convection flow near a vertical plate with Newtonian heating and mass diffusion using Laplace transform. For works related to the unsteady flow of generalized Casson fluid with fractional derivative, the readers are recommended to take a look at [6]. For papers in the same directions of research as addressed in our paper, see in [30-33].

This paper's question is to determine the exact solutions of the fluid model using the Laplace transforms. The advantages of this paper are as follows: at first, the solutions will be expressed using well-known functions as Gaussian error function, Mittag-Leffler function, Wright function, and $G$-function [29]. Thus, the graphical representations of the dynamics of the exact solutions of the proposed model will be easy to be obtained via MATLAB manipulations. Another advantage of this paper is that some new initial conditions not previously considered in the literature will be used and studied in detail. The influence of the order of the fractional operator will be studied and analyzed. The physical interpretations of the influence of the Caputo derivative will be proposed. The same procedure will be done with the parameters of the model. In other words, the influences of the model parameters in the dynamics will be focused on, analyzed, and interpreted. In our modeling, the influence of the Nusselt number and the Sherwood number will be presented and discussed, too. The fact that the solutions can be obtained via the resolution of the secondorder differential equation is also a significant advantage of the Laplace transform method.

The present paper is structured as follows: In Section 2, the fractional operators and the special functions used in this paper have been recalled. In Section 3, the natural convection flow of an incompressible viscous fluid subject to Newtonian heating and constant mass diffusion model has been proposed using Caputo derivative. In the modeling, velocity, temperature, and concentrations of the fluid have been considered and analyzed for the considered model. In Section 4, the solutions procedure has been proposed via the Laplace transform. In Section 5, we discuss the main findings of the paper via graphical representations. In Section 6, we give the conclusion and the final remarks and discuss the main findings of the present paper and its advantages in the existing literature.

\section{Preliminaries}

This part is devoted to recalling certain fractional operators necessary for our present investigations. As known, there exist many fractional operators such as Riemann-Liouville integral, the Caputo fractional derivative, Riemann-Liouville derivative, derivative with the exponential kernel, derivative with Mittag-Leffler kernel. The use of fractional operators in modeling real-world problems is motivated by the memory effect. Here, we will investigate using the Caputo operator because it satisfies, in particular, the initial conditions which we planned to use. Note that the Riemann-Liouville derivative does not satisfy the initial conditions used in the present investigations. We give the following definitions.

We represent the Riemann-Liouville integral as the following form $[13,14]$ :

$$
I^{\alpha} u(t)=\frac{1}{\Gamma(\alpha)} \int_{0}^{t}(t-s)^{\alpha-1} u(s) d s,
$$

where the function $u:[0,+\infty[\longrightarrow \mathbb{R}$ and the order obeys the condition $\alpha>0$. The function $\Gamma(\ldots)$ denotes the Gamma Euler function.

The Caputo fractional derivative as reported in the literature can be represented as the following form $[12,15]$ :

$$
D_{c}^{\alpha} u(t)=\frac{1}{\Gamma(1-\alpha)} \int_{0}^{t}(t-s)^{-\alpha} u^{\prime}(s) d s,
$$

where the function $u:[0,+\infty[\longrightarrow \mathbb{R}$ and the order is taken in the interval $\alpha \in(0.1)$.

Without losing generalities, we recall the Riemann-Liouville derivative as reported in the literature with the following form $[12,15]$ :

$$
D^{\alpha} u(t)=\frac{1}{\Gamma(1-\alpha)} \frac{d}{d t} \int_{0}^{t} u(s)(t-s)^{-\alpha} d s,
$$

with the condition that $t>0$, the order of the fractional operator satisfies the condition $\alpha \in(0,1)$, and $\Gamma(\ldots)$ represents the Gamma Euler function. 
There exists a recent fractional operator in fractional calculus. We mean the following definition of the Caputo-Fabrizio fractional derivative [18] of the function $u:[0,+\infty[\longrightarrow \mathbb{R}$, of order $\alpha$ in the following term:

$$
D^{\alpha, C F} u(t)=\frac{C F(\alpha)}{1-\alpha} \int_{0}^{t} u^{\prime}(s) \exp \left(-\frac{\alpha}{1-\alpha}(t-s)\right) d s,
$$

with the following condition $t>0$, the order of the fractional operator satisfies $\alpha \in(0,1)$ and $C F($.$) is the normalization$ term and respects the condition $C F(0)=C F(1)=1$. The integral associated with the Caputo-Fabrizio derivative is represented as the following form:

$I^{\alpha, C F} u(t)=\frac{2(1-\alpha)}{(2-\alpha) C F(\alpha)} u(t)+\frac{2 \alpha}{(2-\alpha) C F(\alpha)} \int_{0}^{t} f(s) d s$.

We have the fractional derivative with Mittag-Leffler kernel and its associated integral, seen in the literature particularly in the following paper [17]. The following is the definition of the Atangana-Baleanu derivative of the function $u:[0,+\infty[\longrightarrow \mathbb{R}$, of order $\alpha$ :

$$
D^{\alpha, A B} u(t)=\frac{A B(\alpha)}{1-\alpha} \int_{0}^{t} u^{\prime}(s) E_{\alpha}\left(-\frac{\alpha}{1-\alpha}(t-s)^{\alpha}\right) d s,
$$

with respect to the condition $t>0$, the order of the fractional operator $\alpha \in(0,1)$, and $A B($.$) denotes the normalization$ term and satisfies the condition $A B(0)=A B(1)=1$. The integral associated with the Atangana-Baleanu fractional derivative is represented as follows:

$$
I^{\alpha, A B} u(t)=\frac{1-\alpha}{A B(\alpha)} u(t)+\frac{\alpha}{A B(\alpha)} I^{\alpha} u(t) .
$$

We finish this section by recalling the Laplace transform because this transformation is the key of the present paper. In the following line, we recall the Laplace transform of the Caputo derivative, for the function $u$, and we have the following:

$$
\mathscr{L}\left\{\left(D_{c}^{\alpha} u\right)(t)\right\}=s^{\alpha} \mathscr{L}\{u(t)\}-s^{\alpha-1} u(0),
$$

where the order $\alpha$ satisfies the relationship $\alpha \in(0,1)$. The problem consists of establishing the analytical solutions of the fluid models that need some special functions. We mean the Mittag-Leffler function and the Wright function. They will play an important role in expressing the form of analytical solutions. For the Mittag-Leffler function with three parameters [29], we have the following definition:

$$
E_{\alpha, \beta}(x)=\sum_{k=0}^{\infty} \frac{x^{k}}{\Gamma(\alpha k+\beta)},
$$

where $\alpha>0, \beta \in \mathbb{R}$, and $x \in \mathbb{C}$. Note that when $\alpha=\beta=1$, we have the classical exponential function.

We define the Wright function [29] with three parameters as follows:

$$
\Phi(\beta,-\sigma, x)=\sum_{n=0}^{\infty} \frac{x^{n}}{\Gamma(n+1) \Gamma(\beta-\sigma n)},
$$

where the conditions $\sigma \in(0,1), \beta \in \mathbb{R}$, and $x \in \mathbb{C}$ are satisfied.

\section{Modeling Dynamics of the Fractional Model}

In this part, we propose the constructive equations of the model subject of investigation in this paper. We consider an unsteady free conventional flow of an incompressible viscous fluid over an infinite vertical plate with mass diffusion and chemical reaction [3]. We let the $x$-axis be obtained along with the plate in the vertical direction, and the $y$-axis is taken normal to the plate as presented in the literature $[2,29]$. In the present modeling, the use of the Caputo derivative in the newly added assumption. At the neighborhood of the initial time of the diffusion processes, the temperature is constant at $T_{\infty}$ and the concentration on the plate is fixed to $C_{\infty}$ while plate and fluid are at the rest. We suppose that at $t=0^{+}$, the heat and the mass from the plate to the fluid is raised to a variable temperature $T_{\infty}+\left[T_{w}-\right.$ $\left.T_{\infty}\right]\left[t / t_{0}\right]$ and the concentration level near the plate is considered as $C_{\infty}+\left[C_{w}-C_{\infty}\right]\left[t / t_{0}\right]$. For the rest of the modeling, we use the Boussinesq rules. Furthermore, we consider the Caputo fractional operator. Fick's first and second laws in the context of fractional operators reported in the literature [21] will be applied to get the constructive equations. Note that inspired to the modeling described in the paper [21], the Linear momentum equation with Caputo operator can conventionally be represented by the following differential equation:

$$
\rho \frac{\partial v}{\partial t}=\frac{\partial \epsilon}{\partial x}+g \beta D^{1-\alpha}\left(T-T_{\infty}\right)+g \gamma D^{1-\alpha}\left(C-C_{\infty}\right),
$$

where $\epsilon$ represents the shear stress. We use the Caputo derivative to express the shear stress as the following form:

$$
\epsilon=\nu\left(1+\frac{1}{\beta}\right) D_{t}^{1-\alpha}\left[\frac{\partial v}{\partial x}\right]
$$

The next step is to introduce equations (12) into (11). We obtain the following fractional differential equation:

$$
\begin{aligned}
\rho \frac{\partial v}{\partial t}= & \nu\left(1+\frac{1}{\beta}\right) D_{t}^{1-\alpha}\left[\frac{\partial^{2} v}{\partial x^{2}}\right]+g \beta D^{1-\alpha}\left(T-T_{\infty}\right) \\
& +g \gamma D^{1-\alpha}\left(C-C_{\infty}\right) .
\end{aligned}
$$

Applying the Riemann-Liouville integral $I^{1-\alpha}$ to both sides of equation (13), we arrive at our first fractional differential equation described as follows:

$\rho D_{t}^{\alpha} v=v\left(1+\frac{1}{\beta}\right) \frac{\partial^{2} v}{\partial x^{2}}+g \beta\left(T-T_{\infty}\right)+g \gamma\left(C-C_{\infty}\right)$.

The Fick second law related to the thermal balance equation as reported in the literature [21] can be represented by the following form:

$$
\rho C_{p} \frac{\partial T}{\partial t}=-\frac{\partial q}{\partial x}
$$

where $q$ is the thermal flux and obeys Fick's first law reported with the fractional operator and represented as follows: 


$$
q=-k_{0} D_{t}^{1-\alpha}\left[\frac{\partial T}{\partial x}\right]
$$

We replace equations (16) into (15); then, the temperature distribution considered in the present paper can be represented as follows:

$$
\rho C_{p} \frac{\partial T}{\partial t}=k_{0} \frac{\partial}{\partial x}\left[D_{t}^{1-\alpha}\left[\frac{\partial T}{\partial x}\right]\right] .
$$

We repeat the previous procedure by applying the Caputo operator $I^{1-\alpha}$ to both sides of equation (17). We arrive at our second fractional differential equation satisfied by the temperature distribution and described as follows:

$$
\rho C_{p} D_{t}^{\alpha} T=k_{0} \frac{\partial^{2} T}{\partial x^{2}} .
$$

We now give the constructive equation in which the concentration obeys in our modeling. The diffusion equation can be expressed using the Riemann-Liouville integral as follows:

$$
\frac{\partial C}{\partial t}=-\frac{\partial J}{\partial x}-K D_{t}^{1-\alpha}\left(C-C_{\infty}\right)
$$

where $J$ satisfies the molecular diffusion equation given by Fick's first law represented as follows:

$$
J=-d D_{t}^{1-\alpha}\left[\frac{\partial C}{\partial x}\right]
$$

Replacing equation (20) into (19), then the concentration distribution in our present modeling satisfies the following equation:

$$
D_{t}^{\alpha} C=d \frac{\partial^{2} C}{\partial x^{2}}-K\left(C-C_{\infty}\right)
$$

Therefore, the constructive equations of the present work are assigned in the following equations:

$$
\begin{aligned}
\rho D_{t}^{\alpha} v= & \nu\left(1+\frac{1}{\beta}\right) \frac{\partial^{2} v}{\partial x^{2}}+g \beta\left(T-T_{\infty}\right) \\
& +g \gamma\left(C-C_{\infty}\right), \\
\rho C_{p} D_{t}^{\alpha} T= & k_{0} \frac{\partial^{2} T}{\partial x^{2}}, \\
D_{t}^{\alpha} C= & d \frac{\partial^{2} C}{\partial x^{2}}-K\left(C-C_{\infty}\right) .
\end{aligned}
$$

The initial and boundaries conditions are summarized in the following lines, and we have

$$
\begin{aligned}
& v(x, 0)=0, T(x, 0)=T_{\infty}, C(x, 0)=C_{\infty}, \\
& T(0, t)=0, T(0, t)=T_{\infty}+\left[T_{w}-T_{\infty}\right]\left[\frac{t}{t_{0}}\right],
\end{aligned}
$$

$C(0, t)=C_{\infty}+\left[C_{w}-C_{\infty}\right]\left[\frac{t}{t_{0}}\right], v(x, t) \longrightarrow 0$ as $x \longrightarrow \infty$.

Furthermore, we consider that

$$
v \longrightarrow 0, T \longrightarrow T_{\infty}, C \longrightarrow C_{\infty} \text { as } x \longrightarrow \infty \text {. }
$$

To simplify the previous model for helpful manipulations, we will try to introduce dimensionless variables. Here, in our modeling, we set the dimensionless variables [29] as follows:

$$
\begin{aligned}
x^{*} & =\frac{x}{v} t^{*}=\frac{t}{v} v^{*}=\frac{v}{v}, \\
\phi^{*} & =\frac{T-T_{\infty}}{T_{w}-T_{\infty}} \theta^{*}=\frac{C-C_{\infty}}{C_{w}-C_{\infty}}, \\
K^{*} & =\frac{v}{g k_{0}} K .
\end{aligned}
$$

Note that the rest of the modeling follows [29]. Plugging equations (29) into (22) by removing the " $*$ " and multiplying by $1 / \rho$, we have the first constructive equation of our fluid model related to the velocity described as follows:

$$
D_{t}^{\alpha} v=\left(1+\frac{1}{\beta}\right) \frac{\partial^{2} v}{\partial x^{2}}+G r \phi+G m \theta
$$

where $G r$ and $G m$ are defined in the next lines. Replacing equations (29) into (23) by removing the $" *$ " and divising by $\rho C_{p}$, we have the second constructive equation of our fluid model related to the temperature described as follows:

$$
D_{t}^{\alpha} \phi=\frac{1}{\operatorname{Pr}} \frac{\partial^{2} \phi}{\partial x^{2}} .
$$

where Pr is defined in the next lines. Plugging (29) into (24) by removing the $\| * "$, we have the third constructive equation of our fluid model related to the concentration described as follows:

$$
D_{t}^{\alpha} \theta=\frac{1}{S c} \frac{\partial^{2} \theta}{\partial x^{2}}-K \theta,
$$

where $S c$ is defined in the next lines. The velocity, temperature, and concentration according to dimensionless variables at initial time $t=0$ are summarized as follows:

$$
v(x, 0)=\phi(x, 0)=\theta(x, 0)=0 .
$$

Note that we consider velocity $v$, temperature $\phi$, and concentration $\theta$ satisfying the boundary conditions represented in the following relationships:

$$
\begin{gathered}
v(0, t)=0, \\
\phi(0, t)=t=\theta(0, t) .
\end{gathered}
$$

In our previous constructive equations, we consider that the Prandtl number, thermal Grashof number, mass Grashof 
number, and chemical reaction parameter are defined, respectively, as follows:

$$
\begin{aligned}
& \operatorname{Pr}=\frac{\mu C_{p}}{k_{0}}, \quad G r=v g \beta\left(T_{w}-T_{\infty}\right), \\
& S c=\frac{\nu}{d}, \quad G m=\operatorname{\gamma g} v\left(C_{w}-C_{\infty}\right) .
\end{aligned}
$$

To complete the modeling, we give tables of nomenclature where all parameters described in this paper have been summarized for more comprehension (Tables 1 and 2).

\section{Dynamics Approaches}

This section provides the analytical solutions of the equations established in our modeling section. The procedure to obtain the solutions starts with the second and the third equation and finishes with the first equation. This procedure is adopted because the first equation of the constructive equation (30) contains solutions of the second and last equations of models (31) and (32). In this section, the technique will use the Laplace transform and the Mittag-Leffler function, Gaussian error function, and Wright function. These functions will play an important role in expressing the forms of the analytical solutions.

4.1. Dynamics Approaches of the Concentration. We begin with the fractional diffusion equation with the reaction term expressed in equation (32) under the initial and boundaries conditions (33)-(35). See the following fractional differential equation:

$$
D_{\tau}^{\alpha} \theta=\frac{1}{S c} \frac{\partial^{2} \theta}{\partial x^{2}}-K \theta .
$$

Its initial and boundary conditions are described in the following equations:

$$
\begin{aligned}
& \theta(x, 0)=0, \\
& \theta(0, \tau)=t .
\end{aligned}
$$

As previously mentioned, the considered initial and boundary conditions are one of the novelties of the present paper. The solution approach will apply the Laplace transform to both sides of (38). Note that the Laplace transform of the fractional operator will also be used, and we obtain the following relationships:

$$
\begin{aligned}
s^{\alpha} \bar{\theta}-s^{\alpha-1} \theta(0) & =\frac{1}{S c} \frac{\partial^{2} \bar{\theta}}{\partial x^{2}}-K \bar{\theta}, \\
s^{\alpha} \bar{\theta} & =\frac{1}{S c} \frac{\partial^{2} \bar{\theta}}{\partial x^{2}}-K \bar{\theta}, \\
\frac{\partial^{2} \bar{\theta}}{\partial x^{2}}-S c s^{\alpha} \bar{\theta}-S c K \bar{\theta} & =0 .
\end{aligned}
$$

Utilizing the Laplace transform of the initial condition described by $\theta(0, s)=1 / s^{2}$ and solving the second-order
TABLE 1: Nomenclature section.

\begin{tabular}{lc}
\hline Parameters & Descriptions \\
\hline$\rho$ & Density \\
$T$ & Temperature distribution of the fluid \\
$T_{w}$ & Constant temperature \\
$T_{\infty}$ & Ambient fluid temperature \\
$C_{w}$ & Constant concentration level at the plate \\
$C$ & Concentration distribution of the fluid \\
$C_{\infty}$ & Ambient fluid concentration \\
$\nu$ & Kinematic viscosity \\
$\mu$ & Dynamic viscosity \\
$K$ & Chemical reaction parameter \\
$\beta$ & Volumetric coefficient of thermal expansion \\
\hline
\end{tabular}

TABle 2: Nomenclature section.

\begin{tabular}{lc}
\hline Parameters & Descriptions \\
\hline$k_{0}$ & Thermal conductivity of the fluid \\
$\rho$ & Density of the fluid \\
$C_{p}$ & Heat at a constant pressure \\
$g$ & Acceleration constant \\
$\lambda$ & Thermal conductivity \\
$d$ & Mass diffusivity \\
$G m$ & Mass Grashof number \\
$G r$ & Thermal Grashof number \\
$\mathrm{Pr}$ & Prandtl number \\
$S c$ & Schmidt number \\
\hline
\end{tabular}

differential equation (41) with the classical mathematical method, the exact analytical solution is given as follows:

$$
\bar{\theta}(x, s)=\frac{s^{\alpha}+K}{s^{2}} \frac{\exp \left[-x \sqrt{S c\left(s^{\alpha}+K\right)}\right]}{s^{\alpha}+K} .
$$

We can observe that in (42), we have a product of two Laplace transform functions. Using convolution rule, the analytical solution of (38) follows the form described by the relationship

$$
\theta(x, t)=\int_{0}^{\infty} f(x, u) g(t) d u,
$$

where, in particular, function $f$ is represented as follows:

$$
f(x, u)=\exp (-K u) \operatorname{erfc}\left(\frac{x \sqrt{S c}}{2 \sqrt{u}}\right),
$$

and function $g$ is a function obtained via integration and the Wright function:

$$
g(t)=\int_{0}^{t} \frac{1}{s}\left[K(t-s)+\frac{(t-s)^{\alpha-1}}{\Gamma(2-\alpha)}\right] W\left(0,-\alpha,-u s^{-\alpha}\right) d s .
$$

It is also important to propose the analytical solution when the order of the fractional operator is $\alpha=1$. To be more clear, we repeat the previous procedure. It will permit some readers to do the same investigation when the integerorder derivative is used. Note that, in integer-order 
derivative, we get the following form of the Laplace transform of the first-order derivative $s \bar{\theta}-\theta(0)$. Using the previous transform, we have the following relationships:

$$
\begin{aligned}
s \bar{\theta}-\theta(0) & =\frac{1}{S c} \frac{\partial^{2} \bar{\theta}}{\partial x^{2}}-K \bar{\theta}, \\
s \bar{\theta} & =\frac{1}{S c} \frac{\partial^{2} \bar{\theta}}{\partial x^{2}}-K \bar{\theta}, \\
\frac{\partial^{2} \bar{\theta}}{\partial x^{2}}-S c(s+K) \bar{\theta} & =0 .
\end{aligned}
$$

We utilize the Laplace transform of the initial condition; that is $\theta(0, s)=1 / s^{2}$. The second-order differential equation (46) with classical method has the following solution:

$$
\bar{\theta}(x, s)=\frac{\exp [-x \sqrt{S c(s+K)}]}{s^{2}} .
$$

The inverse of the Laplace transform according to the second variable can be represented by the following equation:

$$
\begin{aligned}
\theta(x, t)= & \frac{1}{2}\left[\left(t-\frac{x S c}{2 \sqrt{K}}\right) \exp (-x \sqrt{K S c}) \operatorname{ercf}\left(\frac{x \sqrt{S c}}{2 \sqrt{t}}-\sqrt{K t}\right)\right] \\
& +\frac{1}{2}\left[\left(t+\frac{x S c}{2 \sqrt{K}}\right) \exp (x \sqrt{K S c}) \operatorname{ercf}\left(\frac{x \sqrt{S c}}{2 \sqrt{t}}+\sqrt{K t}\right)\right] .
\end{aligned}
$$

We finish this part by focusing on a special case that can be obtained when the $\alpha=1$ and $K=0 . K=0$ means the chemical reaction parameter is null. The procedure of the solution is similar to the integer-order version. After calculation, we have the inverse as follows:

$$
\bar{\theta}(x, s)=\frac{\exp [-x \sqrt{S c s}]}{s^{2}} .
$$

Applying the inverse of the Laplace transform of the previous function (49), we obtain the following relationship:

$$
\theta(x, t)=\left(\frac{x^{2} S c}{2}+t\right) \operatorname{erfc}\left(\frac{x \sqrt{S c}}{2 \sqrt{t}}\right)-\frac{x \sqrt{S c t}}{2 \sqrt{\pi}} \exp \left(-\frac{x^{2} S c}{4 t}\right)
$$

4.2. Dynamics Approaches of the Temperature. In this part, we try to determine the analytical solution of the fractional diffusion equation presented in (31). To be more clear, we recall the fractional differential (31), the problem is defined as follows:

$$
D_{\tau}^{\alpha} \phi=\frac{1}{\operatorname{Pr}} \frac{\partial^{2} \phi}{\partial x^{2}}
$$

and its initial and boundary conditions are described by the following equations:

$$
\begin{gathered}
\phi(x, 0)=0, \\
\phi(0, \tau)=t .
\end{gathered}
$$

The procedure of getting the solution does not change. We apply the Laplace transform in equations (51) and (53) and get the following relationships:

$$
\begin{aligned}
s^{\alpha} \bar{\phi}-s^{\alpha-1} \phi(0) & =\frac{1}{\operatorname{Pr}} \frac{\partial^{2} \bar{\phi}}{\partial x^{2}}, \\
s^{\alpha} \bar{\phi} & =\frac{1}{\operatorname{Pr}} \frac{\partial^{2} \bar{\phi}}{\partial x^{2}}, \\
\frac{\partial^{2} \bar{\phi}}{\partial x^{2}}-\operatorname{Pr} s^{\alpha} \bar{\phi} & =0 .
\end{aligned}
$$

Note that the Laplace transform of the initial condition described in (53) is given by $\phi(0, s)=1 / s^{2}$. Combining it with the solution of the second-order differential equation (54), we obtain the following relation:

$$
\bar{\phi}(x, s)=\frac{\exp \left[-x \sqrt{\operatorname{Pr} s^{\alpha}}\right]}{s^{2}} .
$$

The inverse of this Laplace transform can be written using the wright function described in the preliminary section. We have the following exact analytical solution:

$$
\phi(x, t)=t W\left(2,-\alpha / 2,-x \sqrt{\operatorname{Pr}} t^{-\alpha / 2}\right) .
$$

We continue with a special case. Let the order of the fractional operator $\alpha=1$. The Laplace transform is represented as the following forms:

$$
\begin{aligned}
s \bar{\phi}-s \bar{\phi}(0) & =\frac{1}{\operatorname{Pr}} \frac{\partial^{2} \bar{\phi}}{\partial x^{2}}, \\
s \bar{\phi} & =\frac{1}{\operatorname{Pr}} \frac{\partial^{2} \bar{\phi}}{\partial x^{2}}, \\
\frac{\partial^{2} \bar{\phi}}{\partial x^{2}}-\operatorname{Pr} s \bar{\phi} & =0 .
\end{aligned}
$$

The Laplace transform of the initial condition described in (53) gives $\phi(0, s)=1 / s^{2}$. Combining it with the solution of 
the second-order differential equation (57), we obtain the following relation:

$$
\bar{\phi}(x, s)=\frac{\exp [-x \sqrt{\operatorname{Pr} s}]}{s^{2}} .
$$

Applying the inverse of the Laplace transform of the previous function (46), we obtain the following relationship:

$\phi(x, t)=\left(\frac{x^{2} \operatorname{Pr}}{2}+t\right) \operatorname{erfc}\left(\frac{x \sqrt{\operatorname{Pr}}}{2 \sqrt{t}}\right)-\frac{x \sqrt{\operatorname{Prt}}}{2 \sqrt{\pi}} \exp \left(-\frac{x^{2} \operatorname{Pr}}{4 t}\right)$.

4.3. Dynamics Approaches of the Velocity. We propose the velocity dynamics in the model proposed in the modeling section. Here, the procedure does not change, and the Laplace transform will be applied. Let the fractional differential equation be defined by the following equation:

$$
D_{\tau}^{\alpha} v=\left(1+\frac{1}{\beta}\right) \frac{\partial^{2} v}{\partial x^{2}}+G r \phi+G m \theta
$$

and its initial and boundary conditions are described in the following equations:

$$
\begin{aligned}
& v(x, 0)=0, \\
& v(0, \tau)=0 .
\end{aligned}
$$

For the simplification in the computations, we suppose that $\kappa=1+1 / \beta$. The Laplace transform as described in the previous sections will be applied. The particularity of this present step is that the Laplace transforms in equations (60)-(62) will be used. We have the following relationship:

$$
\begin{aligned}
s^{\alpha} \bar{v}-s^{\alpha-1} v(0) & =\kappa \frac{\partial^{2} \bar{v}}{\partial x^{2}}+G r \bar{\theta}+G m \bar{\phi}, \\
s^{\alpha} \bar{v} & =\kappa \frac{\partial^{2} \bar{v}}{\partial x^{2}}+G r \bar{\theta}+G m \bar{\phi}, \\
\frac{\partial^{2} \bar{v}}{\partial x^{2}}-\frac{s^{\alpha}}{\kappa} \bar{v} & =-\frac{G r}{\kappa} \bar{\theta}-\frac{G m_{\bar{\phi}}}{\kappa} .
\end{aligned}
$$

In the resolution of this equation, we need to replace the terms in the second member by their values. Using the function in equations (42) and (55), we have to solve the following second-order differential equation:

$$
\frac{\partial^{2} \bar{v}}{\partial x^{2}}-\frac{s^{\alpha}}{\kappa} \bar{v}=-\frac{G r}{\kappa} \frac{\exp \left[-x \sqrt{\operatorname{Pr} s^{\alpha}}\right]}{s^{2}}-\frac{G m}{\kappa} \frac{\exp \left[-x \sqrt{S c\left(s^{\alpha}+K\right)}\right]}{s^{2}} .
$$

In the resolution of this equation, we need to get the homogenous solution and the particular solution. We first consider the equation defined by

$$
\frac{\partial^{2} \bar{v}}{\partial x^{2}}-\frac{s^{\alpha}}{\kappa} \bar{v}=-\frac{G m}{\kappa} \frac{\exp \left[-x \sqrt{S c\left(s^{\alpha}+K\right)}\right]}{s^{2}} .
$$

Its solution considering the Laplace transform of the initial condition can be represented as the following form:

$$
\bar{v}(x, s)=\bar{a}(x, s)+\bar{b}(x, s)+\bar{c}(x, s)+\bar{d}(x, s),
$$

with the functions defined as

$$
\begin{aligned}
& \bar{a}(x, s)=\frac{G m}{K \kappa S c} \frac{s^{\alpha}}{s^{2}} \frac{\exp \left(-x \sqrt{s^{\alpha} / \kappa}\right)}{s^{\alpha}}, \\
& \bar{b}(x, s)=-\frac{G m s^{\alpha-(2-\alpha)}}{K \kappa S c\left(s^{\alpha}+b_{1}\right)} \frac{\exp \left(-x \sqrt{s^{\alpha} / \kappa}\right)}{s^{\alpha}}, \\
& \bar{c}(x, s)=-\frac{G m}{K \kappa S c} \frac{s^{\alpha}+K}{s^{2}} \frac{\exp \left(-x \sqrt{S c\left(s^{\alpha}+K\right)}\right)}{s^{\alpha}+K}, \\
& \bar{d}(x, s)=\frac{G m s^{\alpha-2}\left(s^{\alpha}+K\right)}{K \kappa S c\left(s^{\alpha}+b_{1}\right)} \frac{\exp \left(-x \sqrt{S c\left(s^{\alpha}+K\right)}\right)}{s^{\alpha}+K} .
\end{aligned}
$$

Note that the constant $b_{1}=K \kappa S c /(\kappa S c-1)$. The analytical solution of the model considered in our paper will be calculated by the inverse of the Laplace transforms of the functions represented in equations (67) to (70). We begin with the first function. We need to invert the function $\bar{a}$ using the convolution rule and have the following:

$$
a(x, t)=\frac{G m}{K \kappa S c} \int_{0}^{t} \frac{(t-\tau)^{1-\alpha}}{\Gamma(2-\alpha)} f_{1}(x, \tau) d \tau,
$$

where, in particular, the functions $f$ is represented by the following form:

$$
f_{1}(x, t)=\int_{0}^{\infty} \operatorname{erfc}\left(\frac{x}{2 \sqrt{\kappa u}}\right) \frac{1}{t} W\left(0,-\alpha,-u t^{\alpha}\right) d u .
$$

We continue with the inverse of the Laplace transform of the function $\bar{b}$ and adopt the same procedure as previously made:

$$
b(x, t)=-\frac{G m}{K \kappa S c} \int_{0}^{t}(t-\tau)^{1-\alpha} E_{\alpha, 2-\alpha}\left[-b_{1}(t-\tau)^{\alpha}\right] f_{1}(x, \tau) d \tau .
$$

We continue now with the inverse of the function $\bar{c}$. Here, we use the procedure adopted in the determination of the concentration. We have the following:

$$
\bar{c}(x, s)=-\frac{G m}{K \kappa S c} \frac{s^{\alpha}+K}{s^{2}} \frac{\exp \left[-x \sqrt{S c\left(s^{\alpha}+K\right)}\right]}{s^{\alpha}+K} .
$$

We can observe that, in equation (74), according to the product of two Laplace transform functions, the analytical form follows the form described by

$$
c(x, t)=-\frac{G m}{K \kappa S c} \int_{0}^{t}\left[k(t-\tau)+\frac{(t-\tau)^{1-\alpha}}{\Gamma(2-\alpha)}\right] f_{2}(x, \tau) d \tau,
$$

where, in particular, function $f_{2}$ is represented by the following form: 
$f_{2}(x, t)=\int_{0}^{\infty} \exp (-K u) \operatorname{erfc}\left(\frac{x \sqrt{S c}}{2 \sqrt{u}}\right) \frac{1}{t} W\left(0,-\alpha,-u t^{\alpha}\right) d u$.

We finish the first part of the inversion by the function $\bar{d}$. We use the same procedure as in the previous parts and get the following analytical form:

$$
d(x, t)=\frac{G m}{K \kappa S c} \int_{0}^{t} g_{1}(t-\tau) f_{2}(x, \tau) d \tau,
$$

where, in particular, function $f_{2}$ is represented by the following form:

$$
f_{2}(x, t)=\int_{0}^{\infty} \exp (-K u) \operatorname{erfc}\left(\frac{x \sqrt{S c}}{2 \sqrt{u}}\right) \frac{1}{t} W\left(0,-\alpha,-u t^{\alpha}\right) d u,
$$

and the function $g$ is a function obtained via integration and the Wright function:

$$
g_{1}(t)=\frac{E_{\alpha, 2-\alpha}\left(-b_{1} t^{\alpha}\right)}{t^{\alpha-1}}+t E_{\alpha, 2}\left(-b_{1} t^{\alpha}\right) .
$$

We now try to solve the second-order differential equation defined by the following relationship:

$$
\frac{\partial^{2} \bar{v}}{\partial x^{2}}-\frac{s^{\alpha}}{\kappa} \bar{v}=-\frac{G r}{\kappa} \frac{\exp \left[-x \sqrt{\operatorname{Pr} s^{\alpha}}\right]}{s^{2}} .
$$

The solution of equation (80) is not difficult to be established and can be represented as the following form:

$$
\bar{v}(x, s)=\bar{m}(x, s)+\bar{m}(x, s),
$$

where the functions $\bar{m}$ and $\bar{n}$ are defined as

$$
\begin{aligned}
& \bar{m}(x, s)=\frac{G r}{\kappa \operatorname{Pr}-1} \frac{1}{s^{2}} \frac{\exp \left(-x \sqrt{s^{\alpha} / \kappa}\right)}{s^{\alpha}}, \\
& \bar{n}(x, s)=-\frac{G r}{\kappa \operatorname{Pr}-1} \frac{1}{s^{2}} \frac{\exp \left(-x \sqrt{\operatorname{Pr} s^{\alpha}}\right)}{s^{\alpha}} .
\end{aligned}
$$

Applying the inverse of the Laplace transform and combining it with the convolution product, the analytical solution of equation (80) can be represented as follows:

$$
\begin{aligned}
v(x, t) & =\frac{G r}{\kappa \operatorname{Pr}-1} \int_{0}^{t}(t-\tau) f_{1}(x, \tau) d \tau \\
& -\frac{G r}{\kappa \operatorname{Pr}-1} \int_{0}^{t}(t-\tau) f_{2}(x, \tau) d \tau,
\end{aligned}
$$

where, in particular, functions $f_{1}$ and $f_{2}$ are represented by the following forms:

$$
\begin{gathered}
f_{1}(x, t)=\int_{0}^{\infty} \operatorname{erfc}\left(\frac{x}{2 \sqrt{\kappa u}}\right) \frac{1}{t} W\left(0,-\alpha,-u t^{\alpha}\right) d u, \\
f_{2}(x, t)=\int_{0}^{\infty} \operatorname{erf} c\left(\frac{x \sqrt{\operatorname{Pr}}}{2 \sqrt{u}}\right) \frac{1}{t} W\left(0,-\alpha,-u t^{\alpha}\right) d u .
\end{gathered}
$$

The analytical solution of the fractional differential equation described in equation (60) can be obtained by combining the exact analytical solution in equations (71)-(77) and equation (83). We have the following:

$$
\begin{aligned}
v(x, t)= & a(x, t)+b(x, t)+c(x, t)+d(x, t) \\
& +m(x, t)+n(x, t) .
\end{aligned}
$$

We finish this part with a special case. In this part, the special case is when the Caputo derivative is equivalent to the integer-order derivative. So, we consider that $\alpha=1$. For more clarity, the process to get the solution is repeated with the new form of the Laplace transform, and we have the following:

$$
\begin{aligned}
s \bar{v}-v(0) & =\kappa \frac{\partial^{2} \bar{v}}{\partial x^{2}}+G r \bar{\theta}+G m \bar{\phi}, \\
s \bar{v} & =\kappa \frac{\partial^{2} \bar{v}}{\partial x^{2}}+G r \bar{\theta}+G m \bar{\phi}, \\
\frac{\partial^{2} \bar{v}}{\partial x^{2}}-\frac{s}{\kappa} \bar{v} & =-\frac{G r}{\kappa} \bar{\theta}-\frac{G m}{\kappa} \bar{\phi}, \\
\frac{\partial^{2} \bar{v}}{\partial x^{2}}-\frac{s}{\kappa} \bar{v} & =-\frac{G r}{\kappa} \frac{\exp [-x \sqrt{P r s}]}{s^{2}} \\
& -\frac{G m}{\kappa} \frac{\exp [-x \sqrt{S c(s+K)}]}{s^{2}} .
\end{aligned}
$$

The resolution of the second-order differential (87) gives the following analytical Laplace solution:

$$
\begin{aligned}
\bar{v}(x, s)= & \bar{a}(x, s)+\bar{b}(x, s)+\bar{c}(x, s)+\bar{d}(x, s) \\
& +\bar{m}(x, s)+\bar{n}(x, s),
\end{aligned}
$$

where the functions are defined as follows:

$$
\begin{aligned}
& \bar{a}(x, s)=\frac{G m}{K \kappa S c} \frac{\exp (-x \sqrt{s / \kappa})}{s^{2}}, \\
& \bar{b}(x, s)=-\frac{G m}{K b_{1} \kappa S c} \frac{\exp (-x \sqrt{s / \kappa})}{s}+\frac{G m}{K b_{1} \kappa S c} \frac{\exp (-x \sqrt{s / \kappa})}{s-b_{2}},
\end{aligned}
$$




$$
\begin{aligned}
& \bar{c}(x, s)=-\frac{G m}{K \kappa S c} \frac{\exp (-x \sqrt{S c(s+K)})}{s^{2}} \\
& \bar{d}(x, s)=-\frac{G m}{K b_{1} \kappa S c} \frac{\exp (-x \sqrt{S c(s+K)})}{s}+\frac{G m}{K b_{1} \kappa S c} \frac{\exp (-x \sqrt{S c(s+K)})}{s-b_{2}} \\
& \bar{m}(x, s)=\frac{G r}{\kappa \operatorname{Pr}-1} \frac{1}{s^{2}} \frac{\exp (-x \sqrt{s / \kappa})}{s} \\
& \bar{n}(x, s)=-\frac{G r}{\kappa \operatorname{Pr}-1} \frac{1}{s^{2}} \frac{\exp (-x \sqrt{\operatorname{Pr} s})}{s}
\end{aligned}
$$

where $b_{2}=K \kappa S c /(1-\kappa S c)$. The analytical solution of $(60)$ is obtained via inverting the Laplace transform of equation (89). We begin with the first function. The inverse of the function $\bar{a}$ is given by the following formula:

$$
a(x, t)=\frac{G m}{K \kappa S c}\left[\left(\frac{x^{2}}{2 \kappa}+t\right) \operatorname{erf} c\left(\frac{x}{2 \sqrt{\kappa t}}\right)-\frac{x \sqrt{t}}{2 \sqrt{\kappa \pi}} \exp \left(-\frac{x^{2}}{4 \kappa t}\right)\right] .
$$

The inverse of the Laplace transform of the function $\bar{b}$ is given by the following formula:

$$
\begin{aligned}
b(x, t)= & -\frac{G m}{K b_{1} \kappa S c} \operatorname{erfc}\left(\frac{x}{2 \sqrt{\kappa t}}\right)+\frac{G m}{K b_{1} \kappa S c} \frac{e^{b_{2} t}}{2}\left[e^{-x \sqrt{b_{2} / \kappa}} \operatorname{erfc}\left(\frac{x}{2 \sqrt{\kappa t}}-\sqrt{b_{2} t}\right)\right] \\
& +\frac{G m}{K b_{1} \kappa S c} \frac{e^{b_{2} t}}{2}\left[e^{x \sqrt{b_{2} / \kappa}} \operatorname{erf} c\left(\frac{x}{2 \sqrt{\kappa t}}+\sqrt{b_{2} t}\right)\right] .
\end{aligned}
$$

The inverse of the Laplace transform of the function $\bar{c}$ is given by the following representation:

$$
\begin{aligned}
c(x, t)= & -\frac{G m}{K \kappa S c} \frac{1}{2}\left[\left(t-\frac{x \sqrt{S c}}{2 \sqrt{K}}\right) e^{-x \sqrt{S c K}} \operatorname{erfc}\left(\frac{x \sqrt{S c}}{2 \sqrt{t}}-\sqrt{K t}\right)\right] \\
& -\frac{G m}{K \kappa S c} \frac{1}{2}\left[\left(t+\frac{x \sqrt{S c}}{2 \sqrt{K}}\right) e^{x \sqrt{S c K}} \operatorname{erfc}\left(\frac{x \sqrt{S c}}{2 \sqrt{t}}+\sqrt{K t}\right)\right] .
\end{aligned}
$$

The inverse of the Laplace transform of the function $\bar{d}$ is given by the following representation:

$$
\begin{aligned}
d(x, t)= & -\frac{G m}{K b_{1} \kappa S c} \frac{1}{2}\left[e^{-x \sqrt{S c K}} \operatorname{erfc}\left(\frac{x \sqrt{S c}}{2 \sqrt{t}}-\sqrt{K t}\right)\right] \\
& -\frac{G m}{K b_{1} \kappa S c} \frac{1}{2}\left[e^{x \sqrt{S c K}} \operatorname{erfc}\left(\frac{x \sqrt{S c}}{2 \sqrt{t}}+\sqrt{K t}\right)\right] \\
& +\frac{G m}{K b_{1} \kappa S c} \frac{e^{b_{2} t}}{2}\left[e^{-x \sqrt{S c\left(b_{2}+K\right)}} \operatorname{erfc}\left(\frac{x \sqrt{S c}}{2 \sqrt{t}}-\sqrt{\left(b_{2}+K\right) t}\right)\right] \\
& +\frac{G m}{K b_{1} \kappa S c} \frac{e^{b_{2} t}}{2}\left[e^{x \sqrt{S c\left(b_{2}+k\right)}} \operatorname{erfc}\left(\frac{x \sqrt{S c}}{2 \sqrt{t}}+\sqrt{\left(b_{2}+K\right) t}\right)\right]
\end{aligned}
$$


For the inverse of the Laplace transform of the function $\bar{m}$ and $\bar{n}$, we use convolution product properties and get the following representations:

$$
\begin{aligned}
& m(x, t)=\frac{G r}{\kappa \operatorname{Pr}-1} \int_{0}^{t}(t-\tau) \operatorname{erf} c\left(\frac{x}{2 \sqrt{\kappa \tau}}\right) d \tau, \\
& n(x, t)=-\frac{G r}{\kappa \operatorname{Pr}-1} \int_{0}^{t}(t-\tau) \operatorname{erf} c\left(\frac{x \sqrt{\operatorname{Pr}}}{2 \sqrt{\tau}}\right) d \tau .
\end{aligned}
$$

In the discussion section, these solutions will be implemented via MATLAB, and the solution will be drawn. The advantages of the analytical solutions are useful and not difficult to be implemented via MATLAB.

The expression of the Nusselt number is obtained after the inverse of the Laplace transform of a certain function, and we use the temperature distribution given by

$$
N u=-\mathscr{L}^{-1}\left[\lim _{x \longrightarrow 0} \frac{\partial \bar{\phi}(x, s)}{\partial x} .\right.
$$

After calculations, the Nusselt number is represented by the following value:

$$
N u=\sqrt{\operatorname{Pr}} \frac{t^{1-\alpha / 2}}{\Gamma(2-\alpha / 2)} .
$$

The formula of the Sherwood number is obtained after the inverse of the Laplace transform of a certain function, and we use the concentration distribution given by

$$
S h=-\mathscr{L}^{-1}\left[\lim _{x \longrightarrow 0} \frac{\partial \bar{\theta}(x, s)}{\partial x}\right] .
$$

This number is obtained as previously mentioned using the inverse of the function represented in equation (103) and expressed via a special function $G($.$) known as the Lor-$ enzo-Hartley function. We have the following value:

$$
S h=\sqrt{S c}\left\{G_{\alpha, \alpha-2,1 / 2}(-K, t)+K G_{\alpha,-2,1 / 2}(-K, t)\right\} .
$$

\section{Results and Interpretations}

This section represents the dynamics using the exact analytical solutions described in the previous section. We will also analyze the impact of the order of the fractional operator. The influence of the mass Grashof number, thermal Grashof number, Prandtl number, Schmidt number will also be discussed and physically interpreted. We will also analyze and discuss the Nusselt number and Sherwood number in the fractional context.

5.1. Dynamics of the Concentration. This section represents the dynamics of the concentration in our model (30)-(32). In Figures 1(a), 1(b), 2(a), and 2(b), we consider $S c=4$ and $S c=10, K=0.06$. In the first part, we focus on the influence of different orders of the Caputo derivative. We represent the concentration of the fluid in Figures 1(a), 1(b), 2(a), and 2(b) versus the variations of the order of the fractional operator.

We now consider analyzing the influence of the Schmidt number $S c$. We work with different values of the order of the
Caputo derivative, and the time is fixed to $\tau=4$ and $K=0.06$. The concentrations are represented in Figures 3(a), 3(b), 4(a), and 4(b).

We notice with Figures 3(a), 3(b), 4(a), and 4(b), when the Schmidt number $S c$ increases the concentration of the considered fluid decreases as well. This fact can be explained physically by the fact that when the Schmidt number and the order of the Caputo derivative increase, they reduce the diffusivity of the fluid. Thus, the concentration of the fluid decreases. The same behaviors can be observed with the chemical reaction parameter. Therefore, this part is omitted.

We finish the analysis of the influence of the parameters by representing the dynamics of the concentration when the chemical reaction parameter $k=0$. If we use the analytical solution presented in equation (50) in the graphical representations, we have Figures 5(a) and 5(b).

In Figures 5(a) and 5(b), we can observe that the influence of the order of the Caputo derivative and Schmidt number does not change.

5.2. Dynamics of the Temperature. The objective of this section is to give graphical representations of the temperature distribution and analyze the influence of the order of the Caputo derivative $\alpha$ and the Prandtl number Pr. Here, we work with equation (56). In this section, we consider different orders of the Caputo derivative and different values of the Prandtl number. We have the following graphical representations (Figures 6(a), 6(b), 7(a), and 7(b)) for the dynamics of our model (30)-(32) considering the temperature.

From Figures 6(a), 6(b), and 7(a), we can observe that as the order of the Caputo derivative increases when the time is fixed to $\tau=5$, the temperature distribution increases as well. We notice the acceleration effect generated by the Caputo derivative on the temperature dynamics of the considered fluid model. From Figure 7(b), we observe that when the Prandtl number increases, the temperature distribution decreases. This behavior is normal because big values of the Prandtl number reduce the diffusivity of the considered fluid too. Thus, the temperature decreases with it increase.

5.3. Dynamics of the Velocity. This section is interesting and we will analyze the impact of all the parameters of the considered model on the velocity dynamics. Using the solution described in equation (87) and the resolution procedure adopted to obtain the analytical form of the velocity, we can notice both the concentration and the temperature impact the velocity, which make this section more interesting. The influence of the mass Grashof number $\mathrm{Gm}$, Thermal Grashof number $G r$, volumetric coefficient of thermal expansion $\beta$, Prandtl number Pr, and Schmidt number $S c$ should be analyzed. In our first case, fix the following values $G r=5, \operatorname{Pr}=5, \kappa=9.5, G m=5, K=1.5$, and $\tau=2$. We have Figures 8(a), 8(b), 9(a), and 9(b) when we work with different orders of the fractional operator.

Firstly, we observe that in Figures 8(a), 8(b), 9(a), and 9(b), when the order of the Caputo operator increases, the velocity of the considered fluid increases as well. This behavior is due to the accumulation of the memory, which 


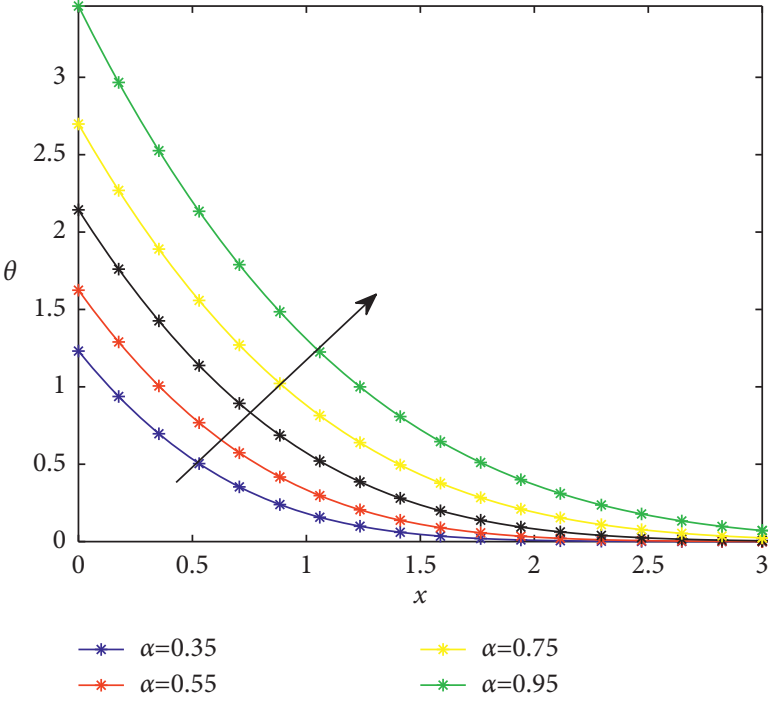

(a)

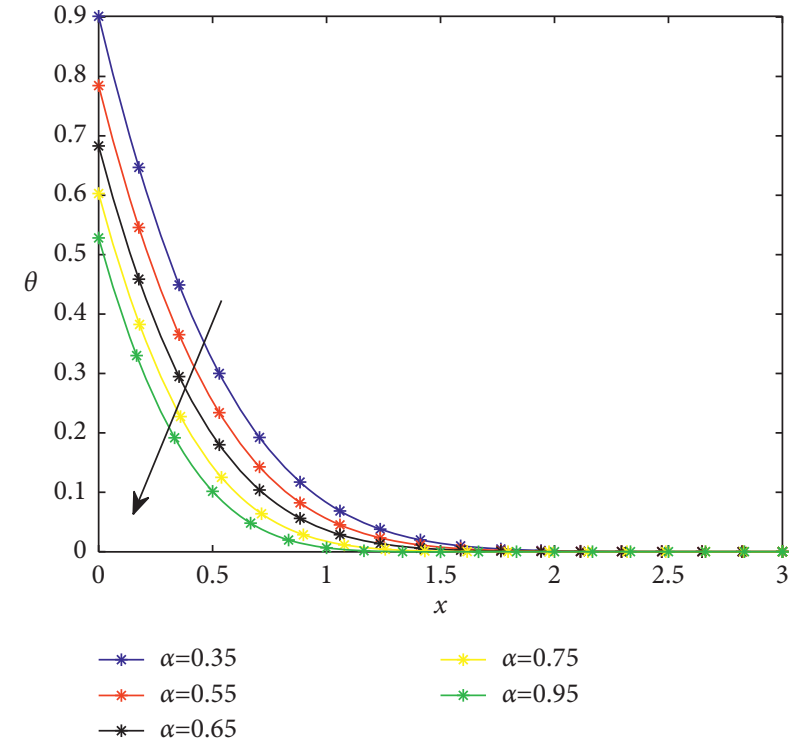

(b)

Figure 1: Concentration for different values of the order $\alpha$. Note. Figures 1(a) and 1(b) are represented with the Schmidt number $S c=4$ and with times $\tau=4$ (a) and $\tau=0.4$ (b).

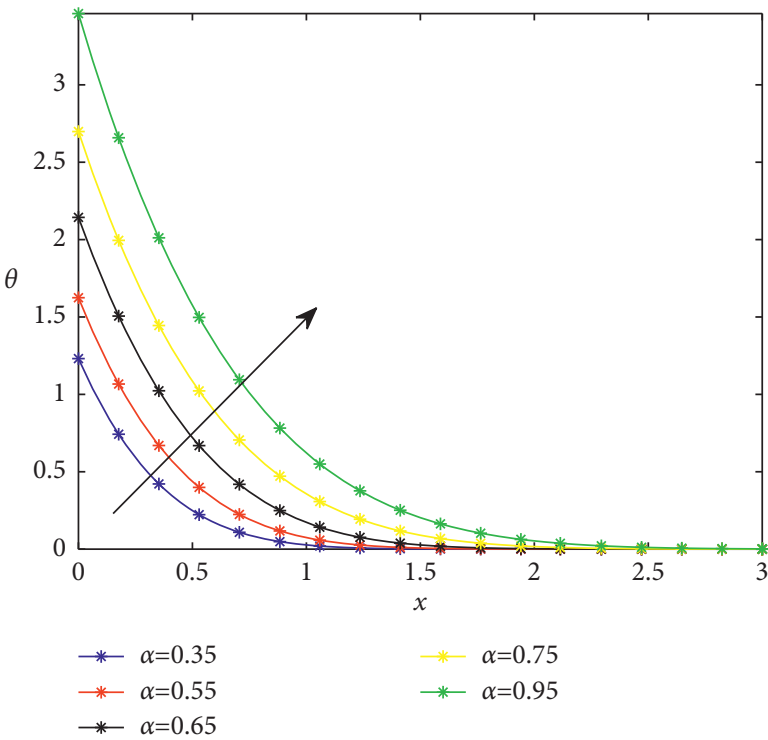

(a)

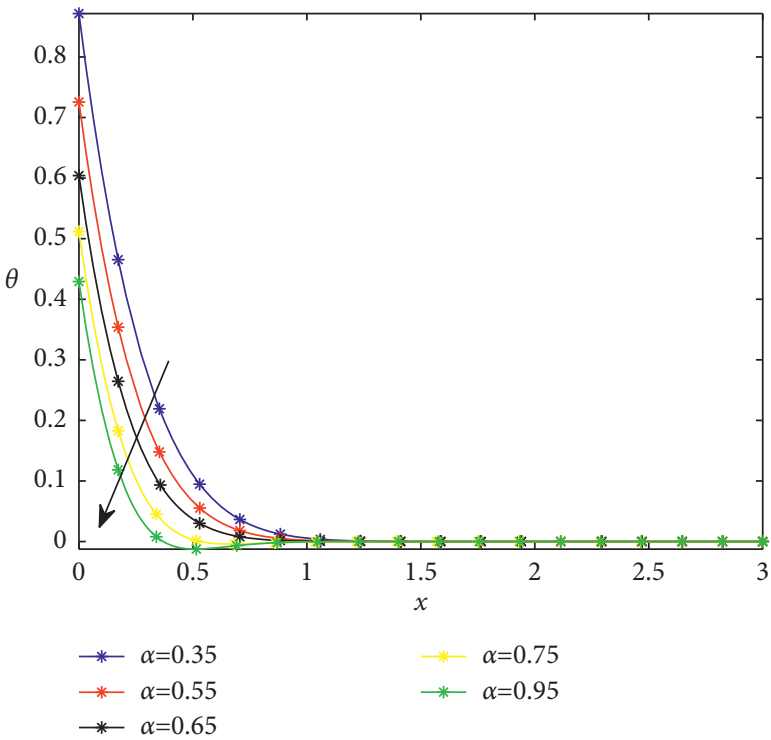

(b)

Figure 2: Concentration for different values of the order $\alpha$. We observe that when $\tau=4$ and the order of the Caputo derivative increases, the concentration of our considered model increases as well. In contrast, when $\tau=0.4$, we notice the increase in the order of the fractional operator generates a decrease in the concentration. The previous behaviors are confirmed when the Schmidt number Sc = 10; see Figures 2(a), and 2(b). Finally, the order of the Caputo derivative has an acceleration or retardation effect that depends on the considered times. These behaviors are explained by the accumulation of the memory effect when the order of the Caputo derivative increases.

causes an acceleration effect in the velocity. The second remark is that the Schmidt number Sc generates a decrease in the velocity when its value increases. The same behaviors are obtained also with the increase of the Prandtl number Pr. These behaviors are already mentioned with temperature and concentration because these values reduce diffusivity. We focus on the influence of the mass Grashof number $G m$.
Before we represent the graphics with the values previously fixed, the difference at this stage is that we take different values for the mass Grashof number $G m$. We have Figures 10(a), 10(b), 11(a), and 11(b).

We use Figures 10(a), 10(b), 11(a), and 11(b) to explain the influence of the mass of the Grashof number. We notice that the increase in this number generates an increase in the 


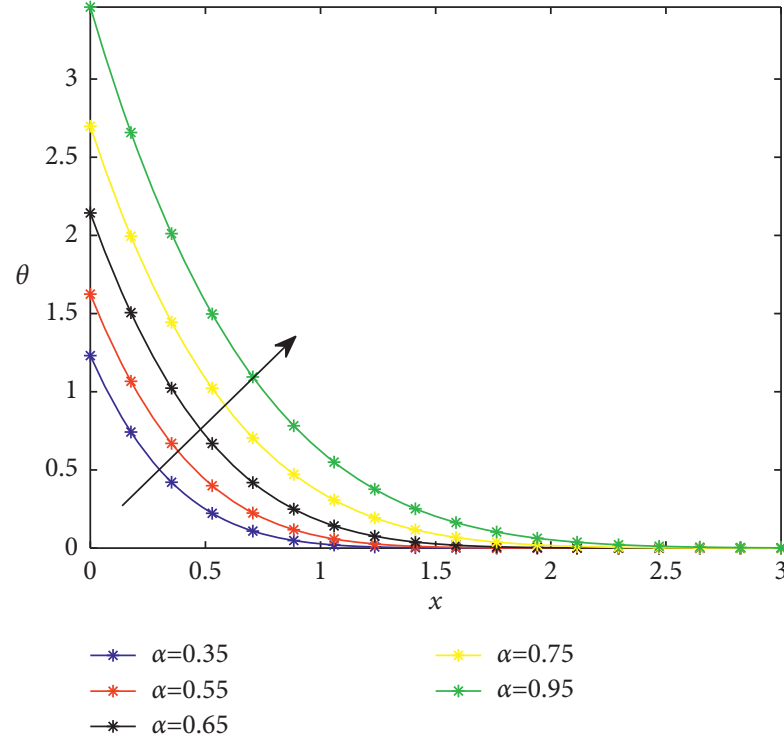

(a)

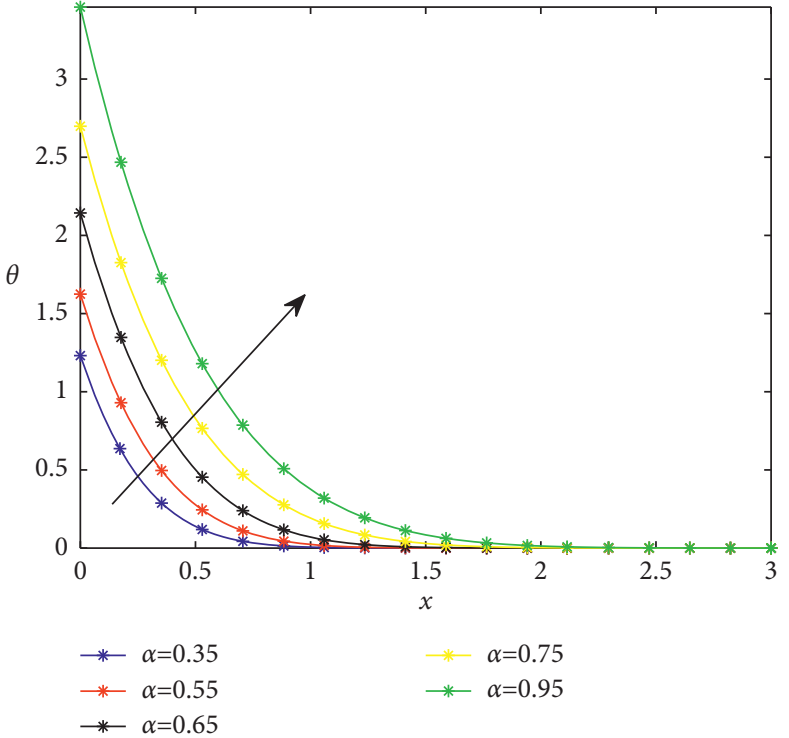

(b)

Figure 3: Concentration for different values of the order $\alpha$ for $S c=10$ (a) and $S c=15$ (b).

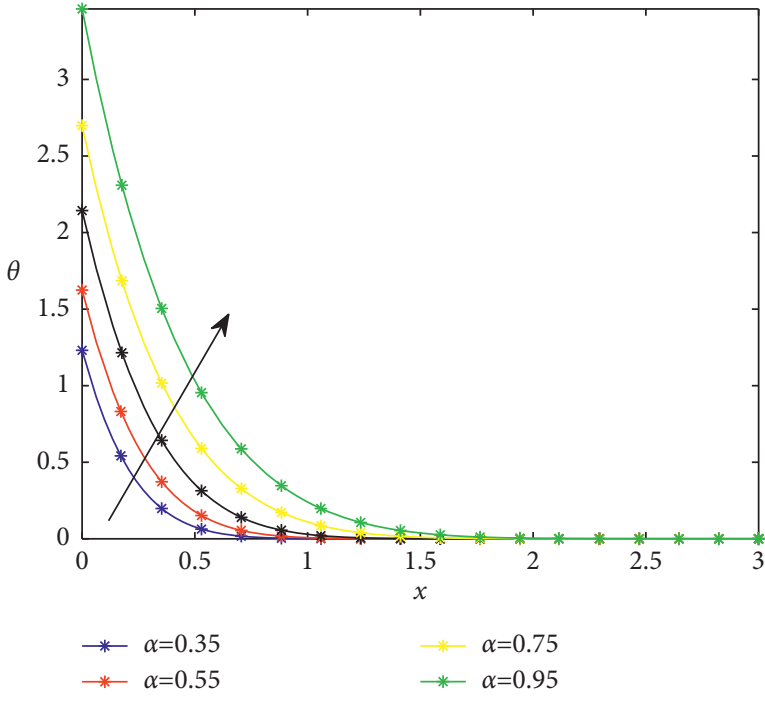

(a)

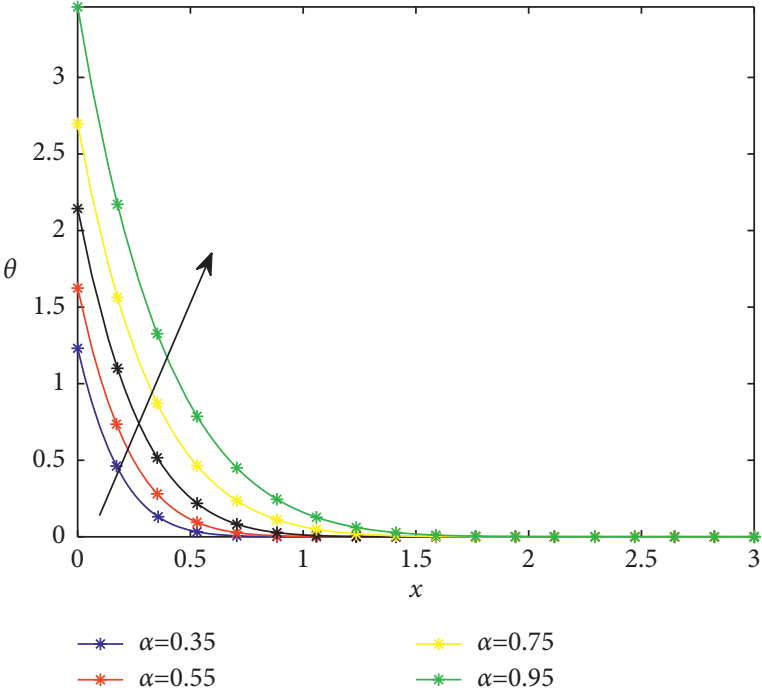

(b)

Figure 4: Concentration for different values of the order $\alpha$ for $S c=20$ (a) and $S c=25$ (b).

velocities. This behavior can be explained physically by the following fact: the mass Grashof number influences the ratio of the species buoyancy force to the viscous hydrodynamic force and its increase generates an increase in the species buoyancy force, which in turn implies an increase in the velocities. The same behavior can be observed with the thermal Grashof number Gm (Figures 12(a), 12(b), 13(a), and 13(b)).

Referring to Figures 12(a), 12(b), 13(a), and 13(b), we observe that the velocities increase when the thermal Grashof number $G r$ increases. These dynamics are because the thermal Grashof number generates an increase in the buoyancy forces, which in turn causes an increase in the momentum boundary layer thickness and then the velocity of the fluid considered in our model.

The comparative studies will be made versus the results in $[21,29]$. As considered in [29], the same model has been considered. For the temperature distribution, the constructive equations described for the temperature in the present paper and [29] are the same, but the initial and boundary conditions differ. The dynamics observed for the temperature in [29] and the present paper in Section 5.2 are similar (Figure 14(a)). The similarity can be explained by the 


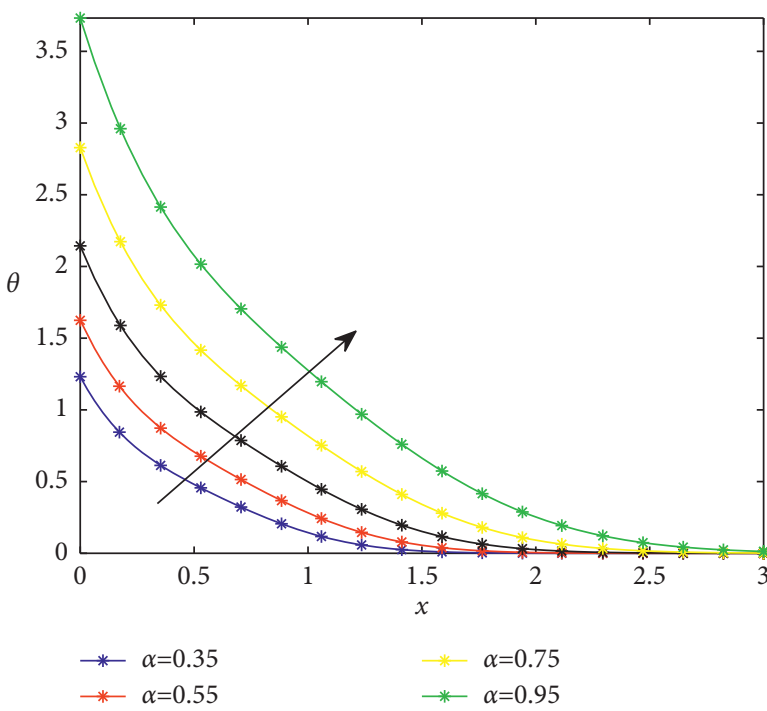

(a)

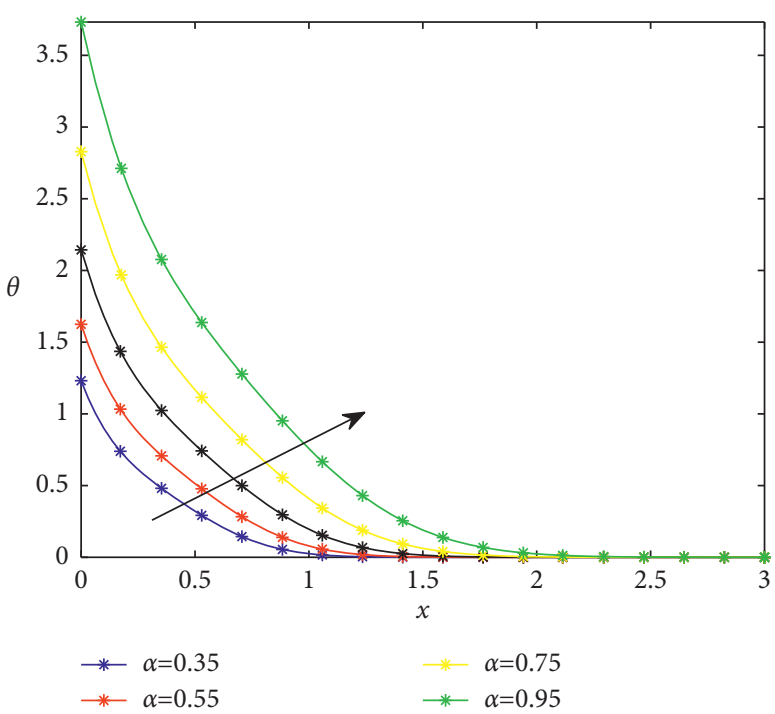

(b)

Figure 5: Concentration for different values of the order $\alpha$ for $S c=10$ (a) and $S c=20$ (b).

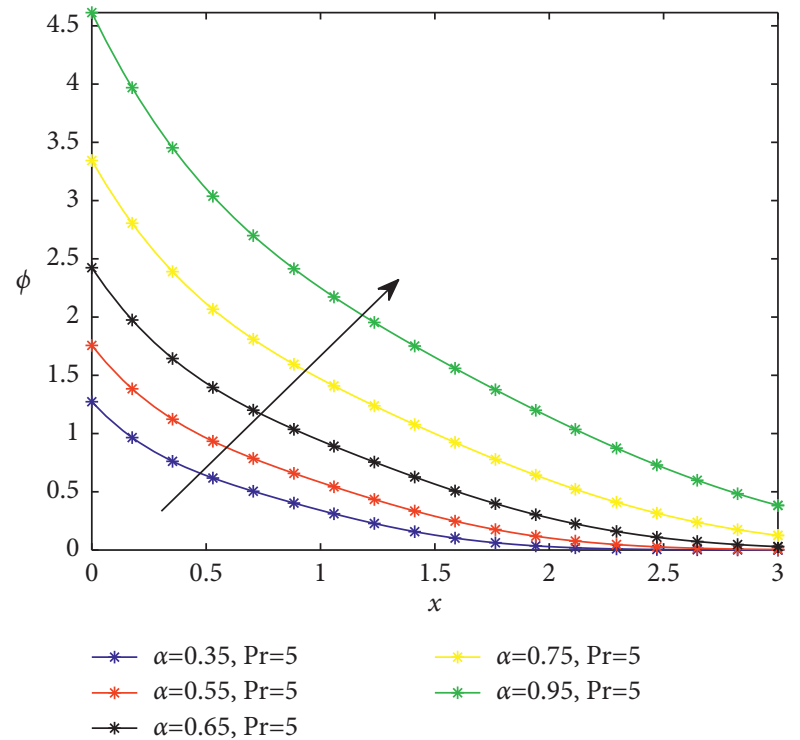

(a)

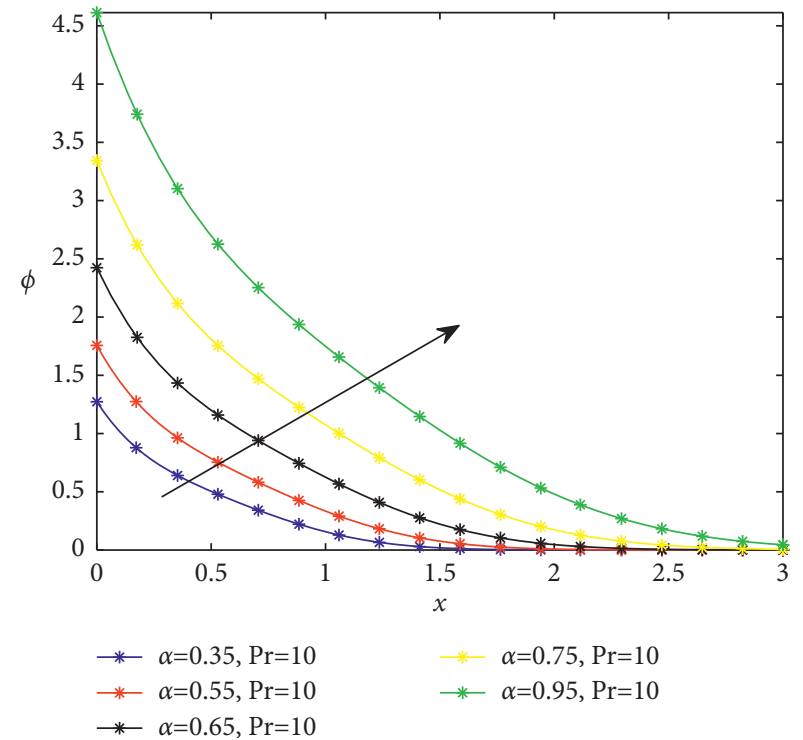

(b)

FIgURE 6: Temperature for different values of order $\alpha$.

fact that our present initial and boundary conditions are a particular case of the boundary and initial conditions considered in [29].

The advantage of our initial condition, in this case, is that it is in a polynomial form, and it is more realistic because it excludes negative value for the derivative of the temperature with respect to the state. In Section 5.1, the concentration has been considered and the constructive equation is the same as the constructive equation for the concentration in [29]. However, the initial conditions are totally different. Here, our initial and boundary condition depends on time, but in [29], they are constant; the consequences are that the dynamics in the figures differ; see Figure 14(b). Note that the difference is caused in this case by the difference in the initial condition. The same initial condition for initial and boundaries conditions have been considered for the velocity. We observe the dynamics of the velocities in [29], and findings in Section 5.3 have the same form, but the values are impacted because the concentrations differ in the paper (Figure 15(a)).

A similar comparison can be made with paper [6].

5.4. Nusselt Number and Sherwood Number. We finish this section by analyzing the influence of the Nusselt number and Sherwood number. These local numbers permit 


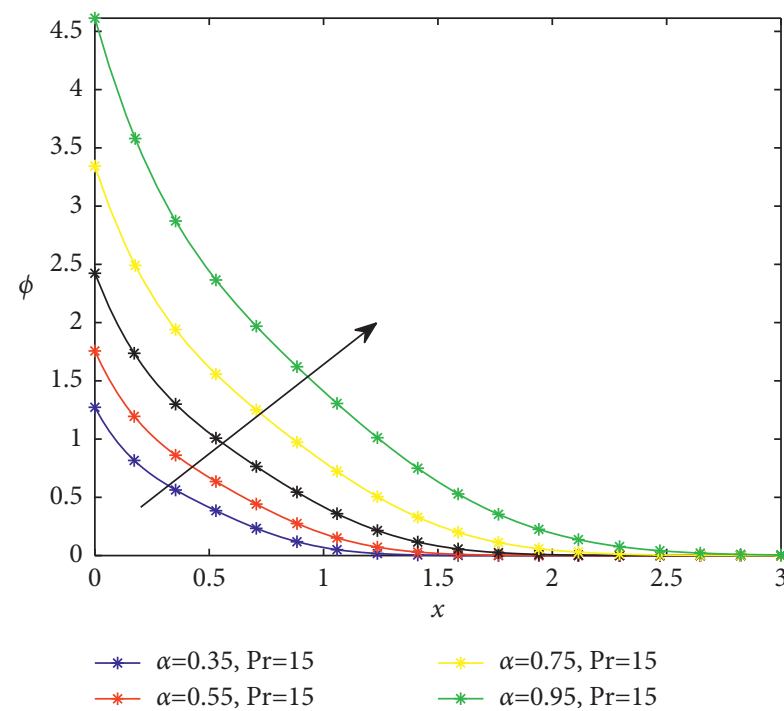

(a)

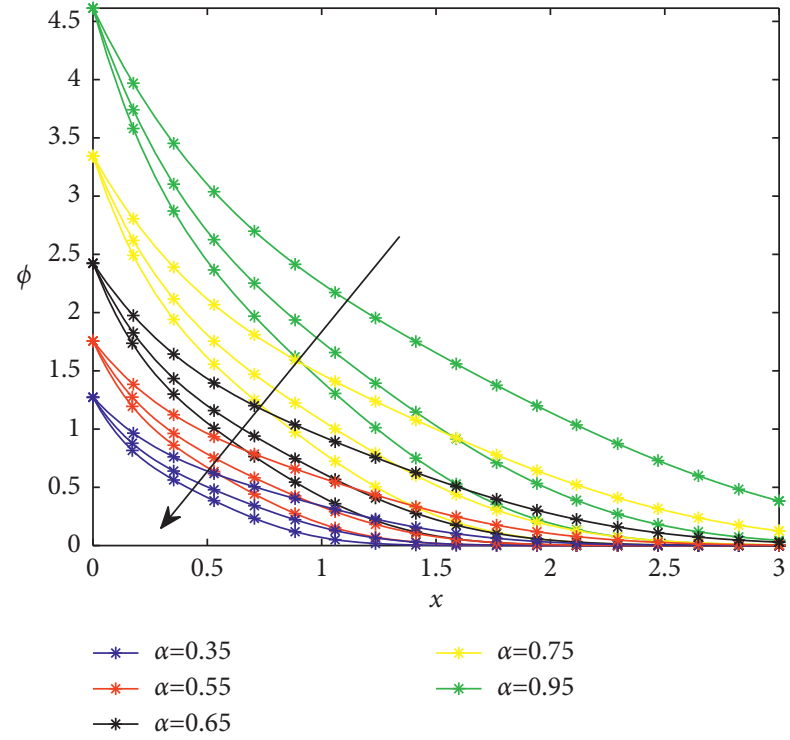

(b)

Figure 7: Temperature for different values of order $\alpha$.

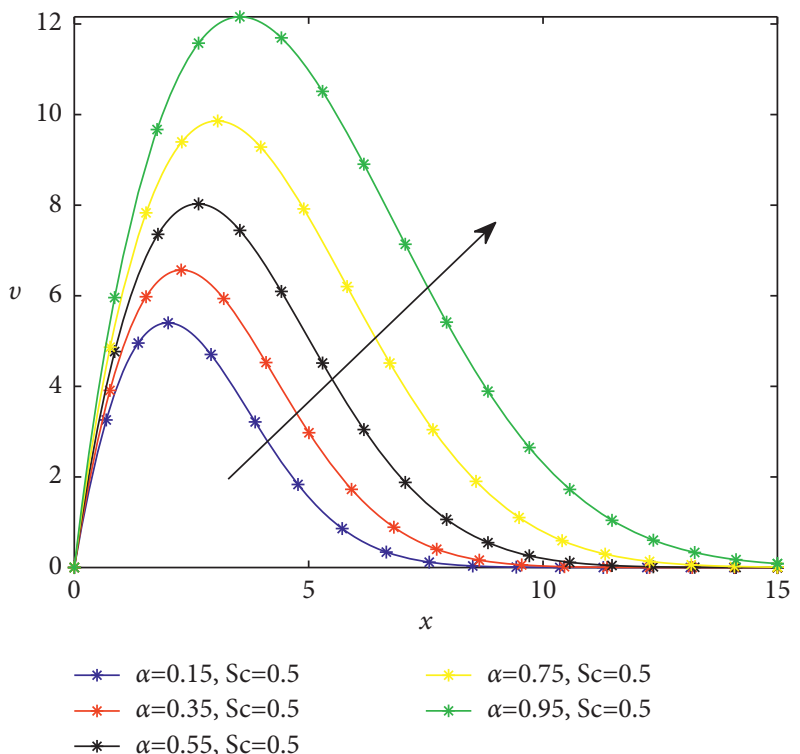

(a)

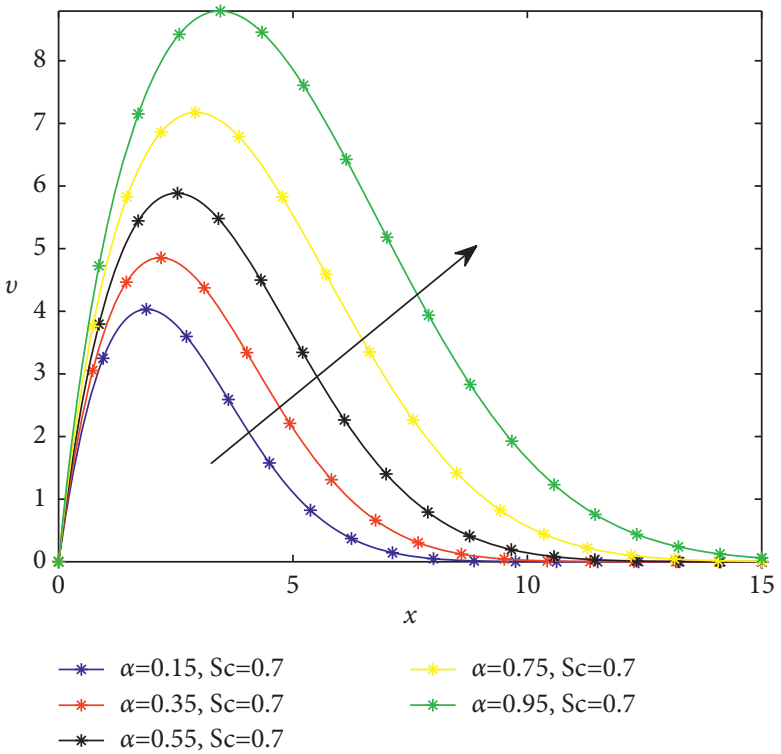

(b)

Figure 8: Velocities for different values of order $\alpha$.

characterizing the mass transfer rate from the plate to the considered fluid. The impact of the Prandtl number and Schmidt number to these local coefficients and the order of the Caputo derivative will be analyzed for more comprehension of our model. Let us consider the Nusselt number with different order of the Caputo derivative; we have Figures 16(a) and 16(b).

We can observe that the variation of the Nusselt number depends strongly on the order of the Caputo derivative. The Nusselt number increases with the increase in the order of the Caputo derivative in the interval $(0,1)$ and decreases with the increase of the order of the operator in the rest of the interval. Figures 16(a) and 16(b) confirm the increase in the Nusselt number when the Prandtl number increases.

We finish with the Sherwood number, which depends on the Schmidt number. The graphical representation is a function of the time and with different values for the order of the fractional operator.

Figures 17(a) and 17(b) confirm the dependence between the Sherwood number, Schmidt number, and order of the fractional operator. Contrary to the Nusselt number, the Sherwood number converges to a small number when the time converges to infinity. 


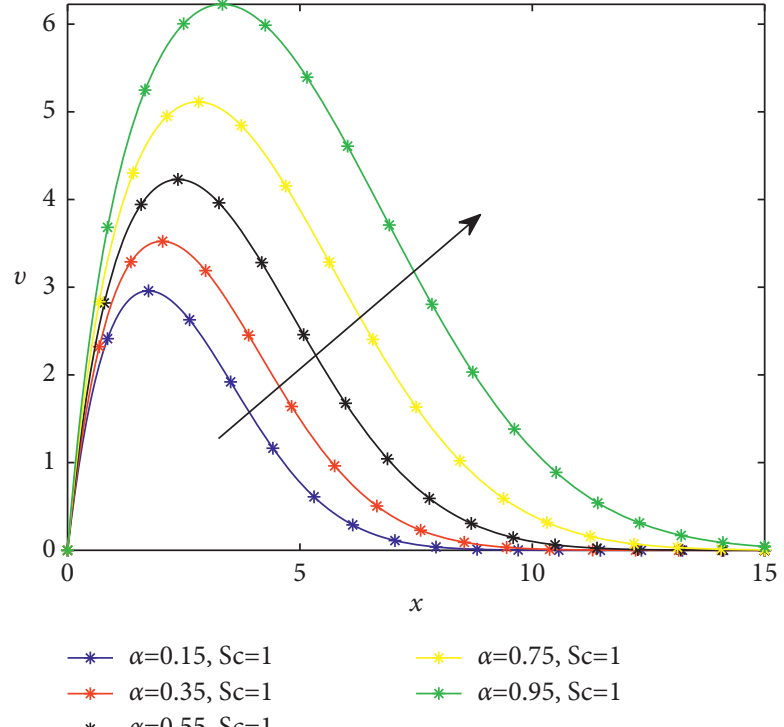

(a)

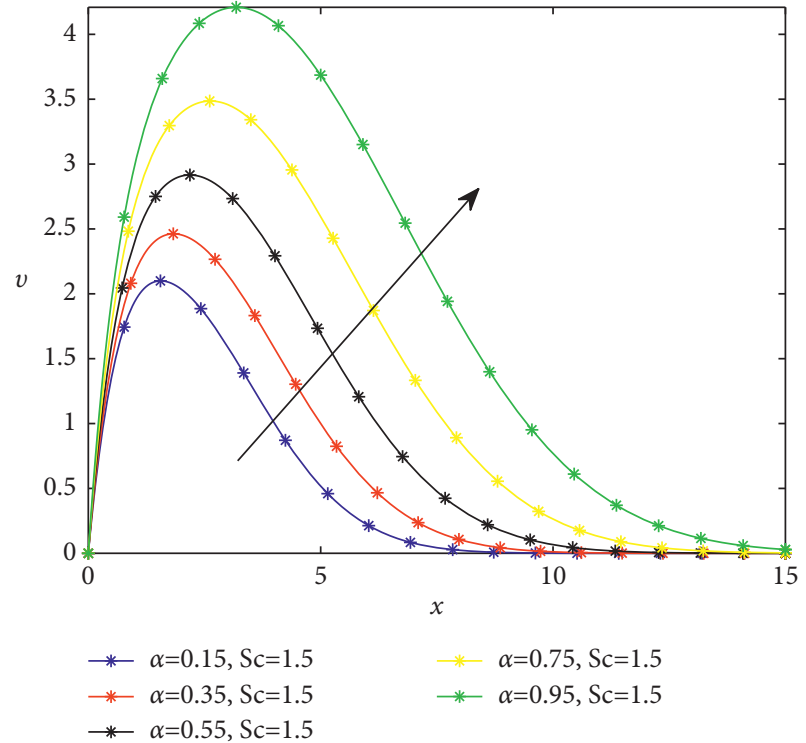

(b)

FIgURE 9: Velocities for different values of order $\alpha$.

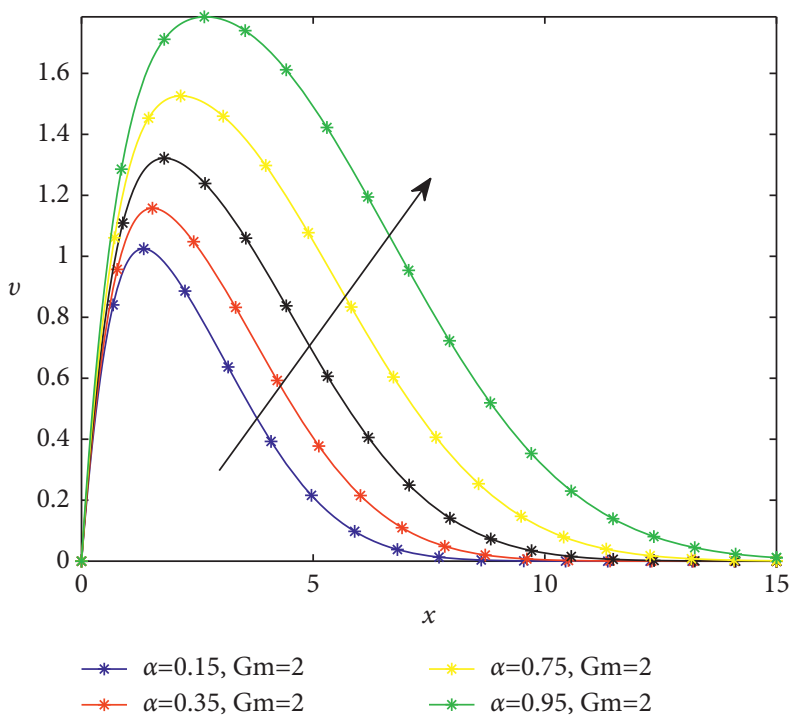

(a)

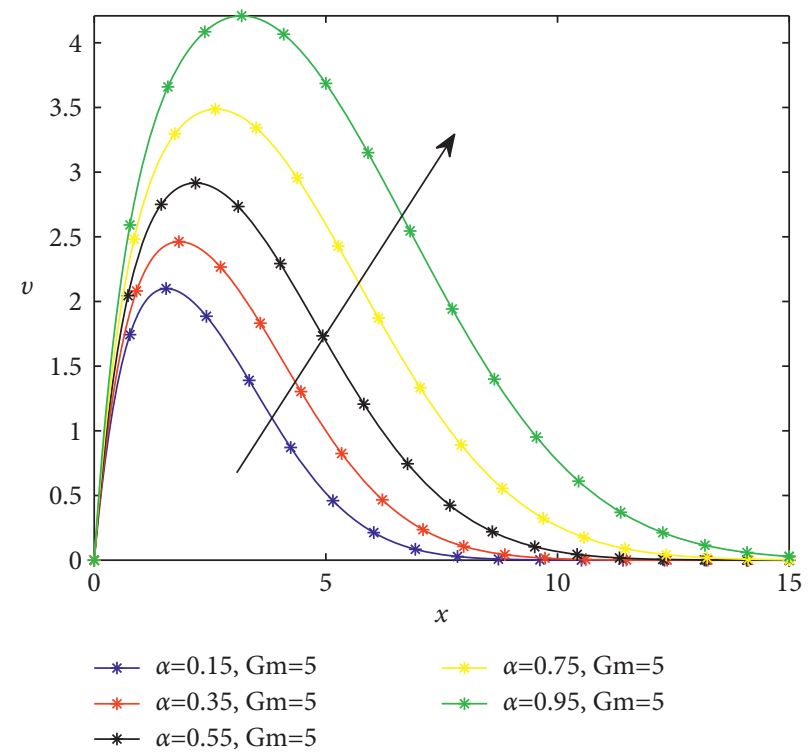

(b)

Figure 10: Velocities for different values of order $\alpha$. 


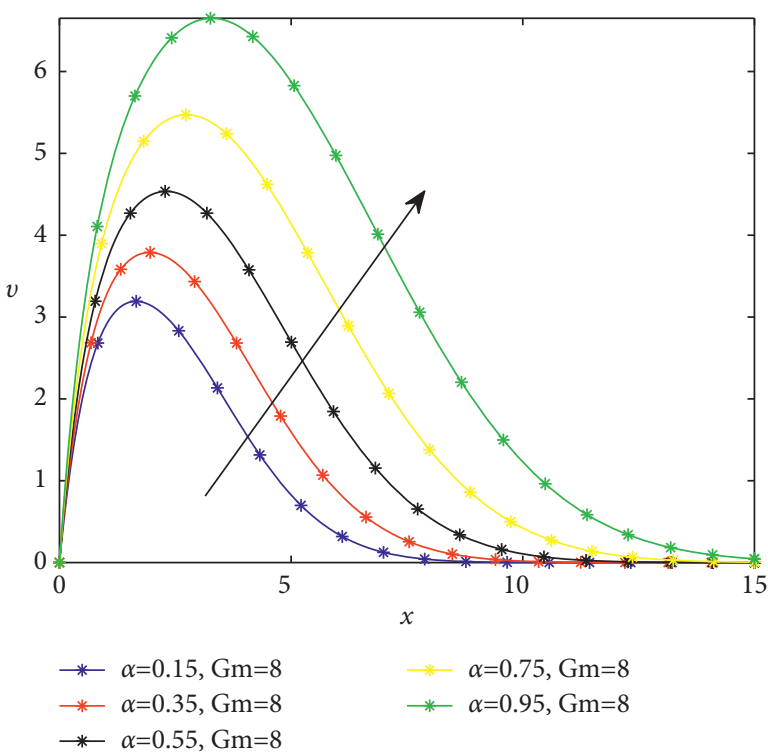

(a)

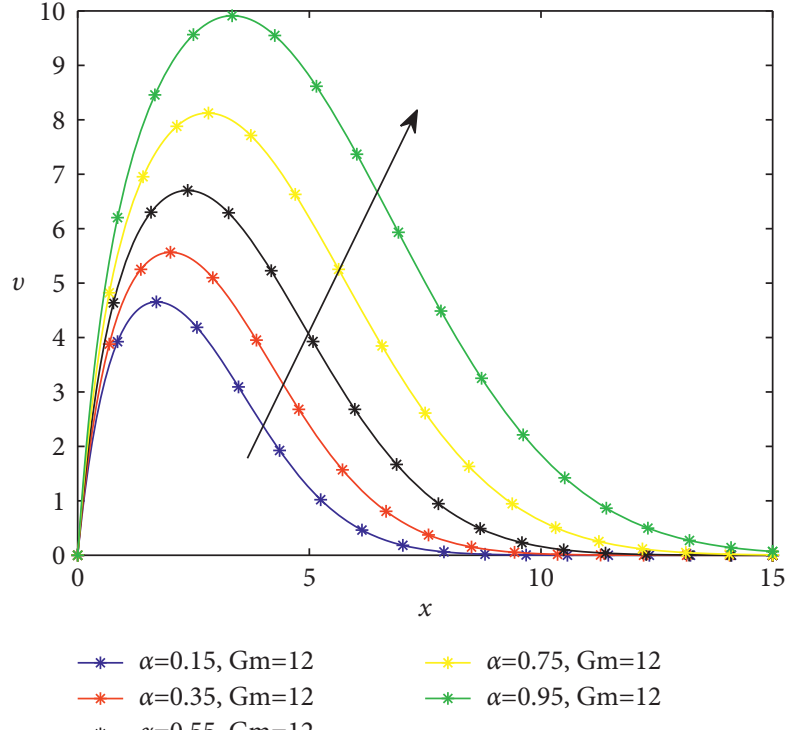

(b)

Figure 11: Velocities for different values of order $\alpha$.

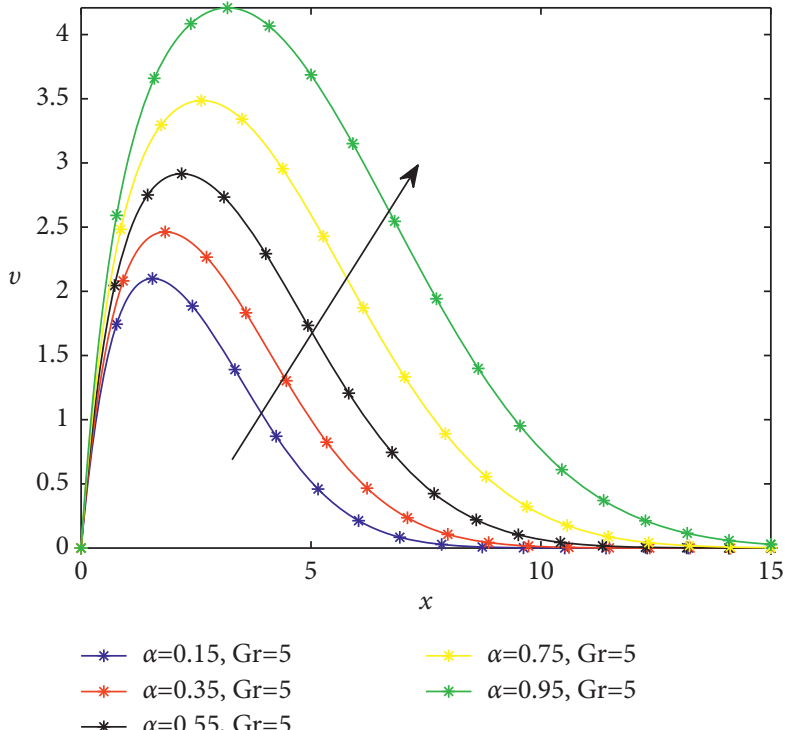

(a)

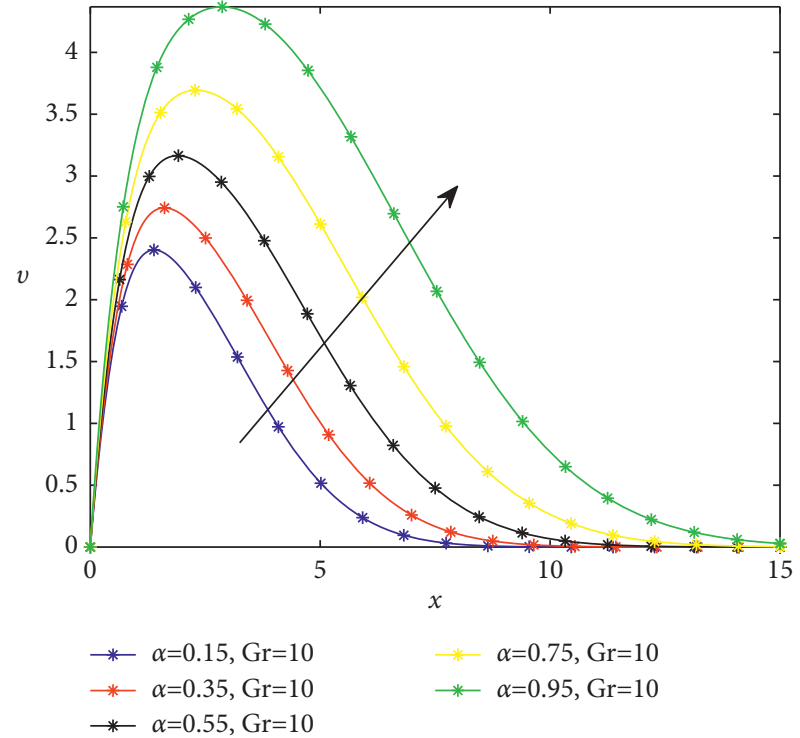

(b)

FIgURE 12: Velocities for different values of order $\alpha$. 


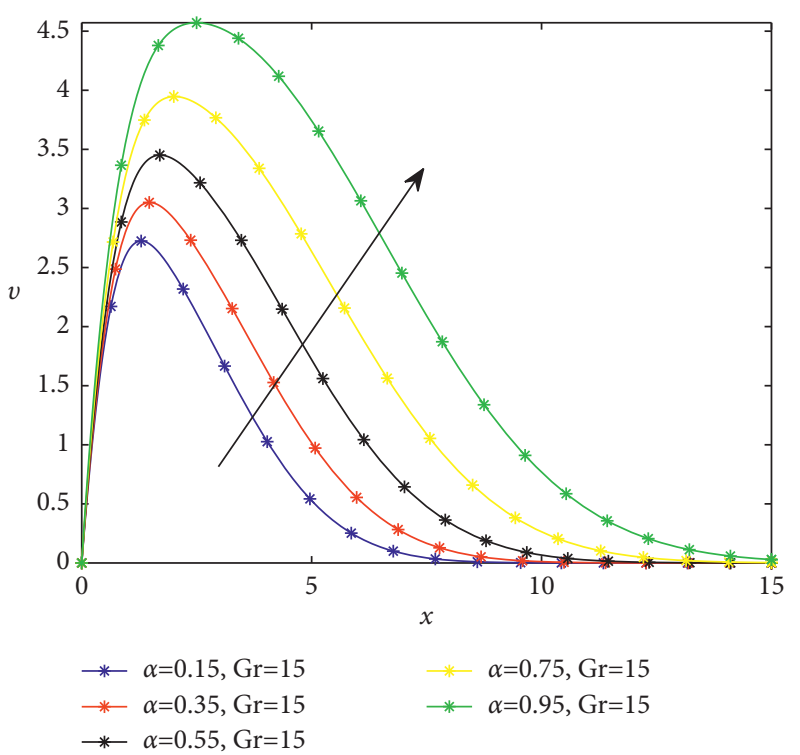

(a)

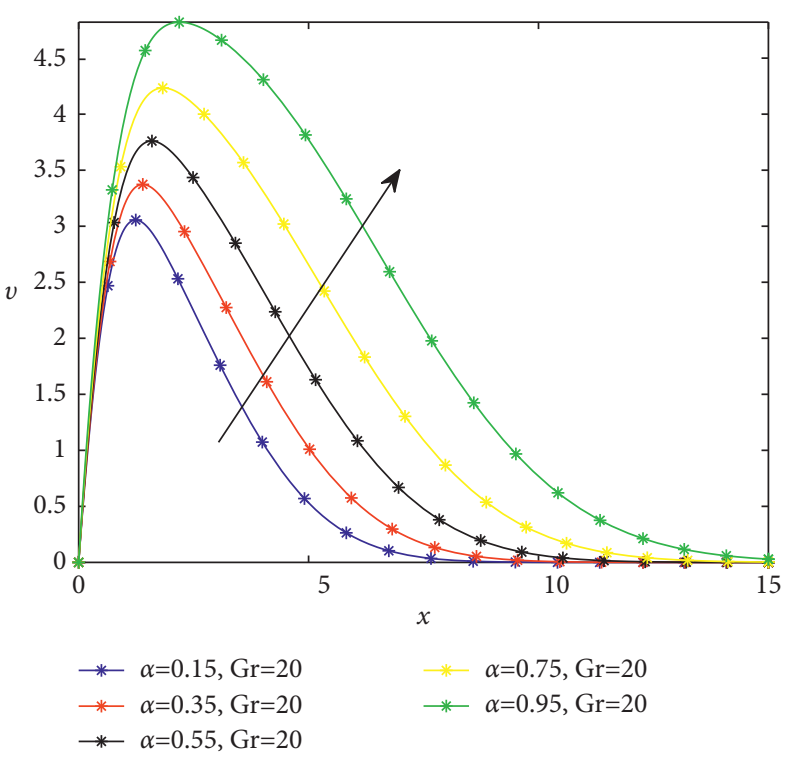

(b)

Figure 13: Velocities for different values of order $\alpha$.

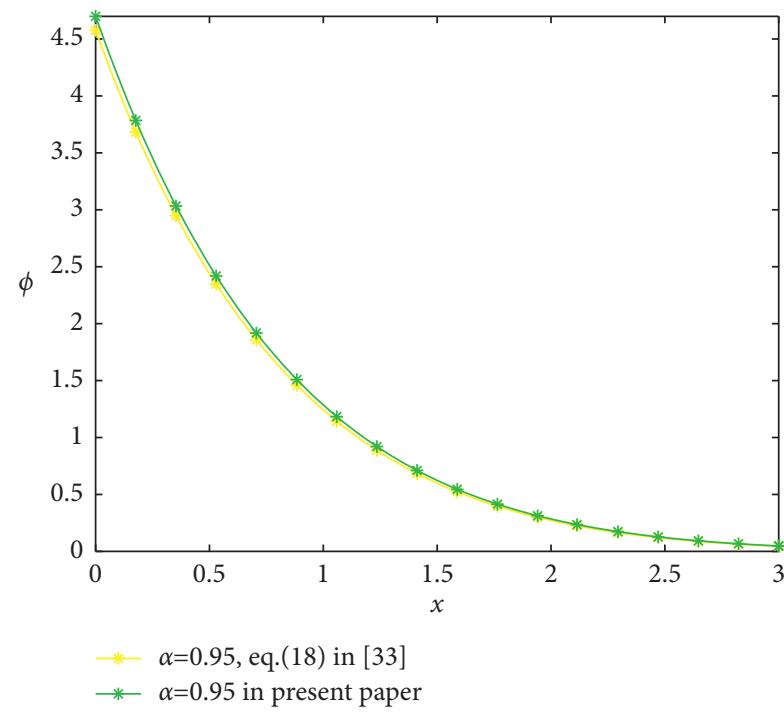

(a)

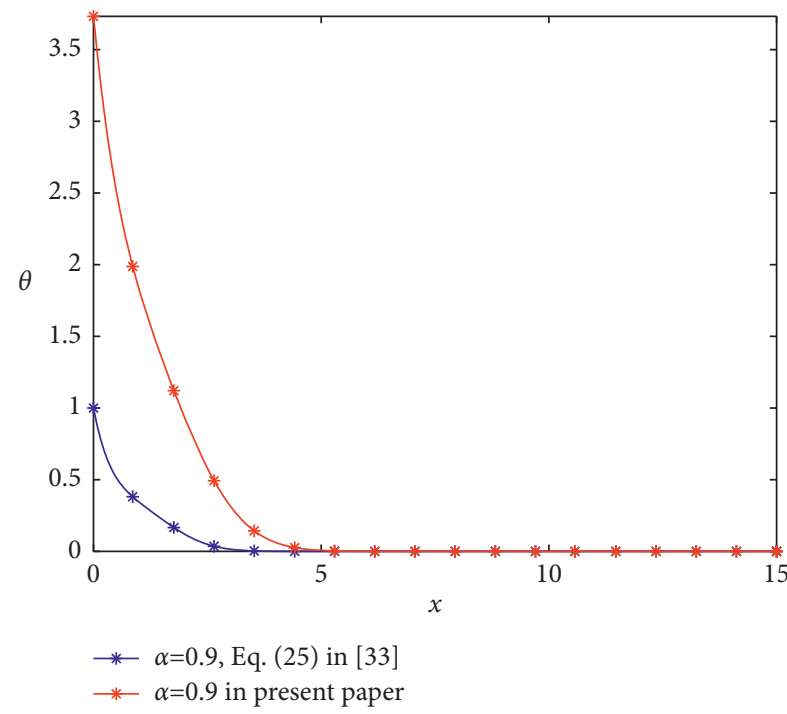

(b)

FIGURE 14: Comparative studies concerning temperature and concentration distribution. 


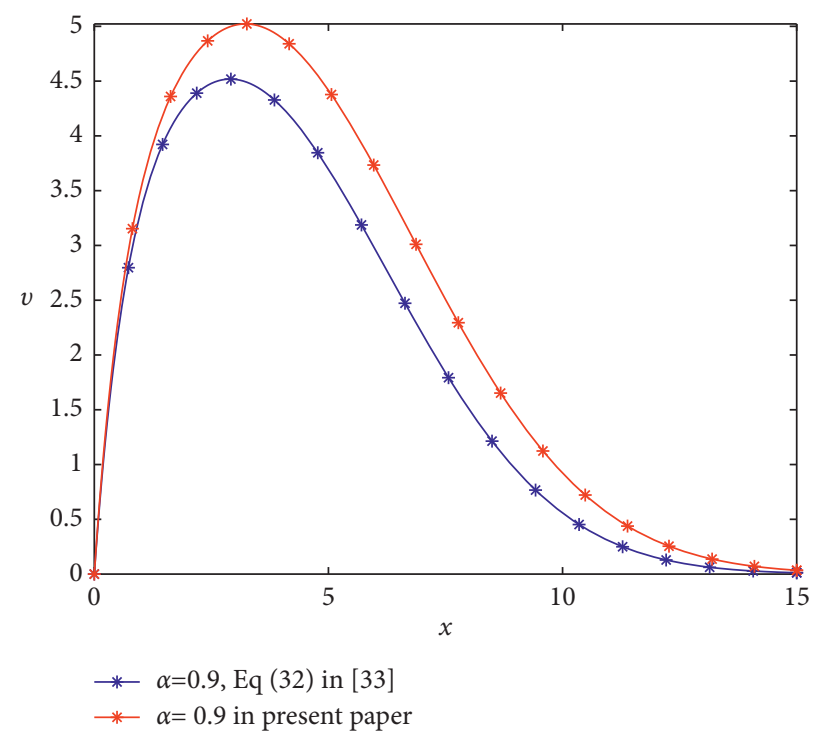

FIgURE 15: Comparative studies concerning the velocity distribution.

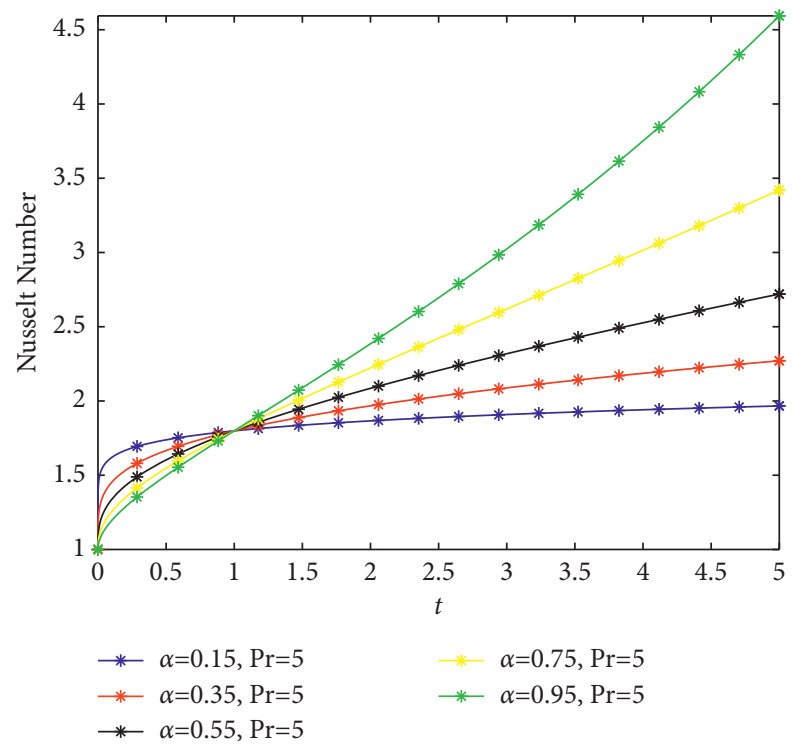

(a)

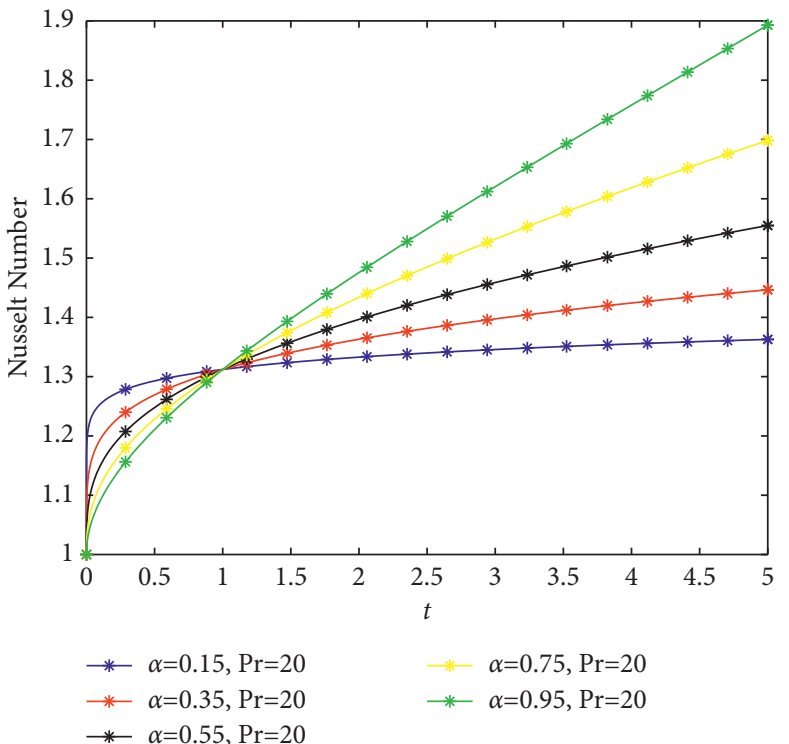

(b)

FIGURE 16: Nusselt number for different values of order $\alpha$. 


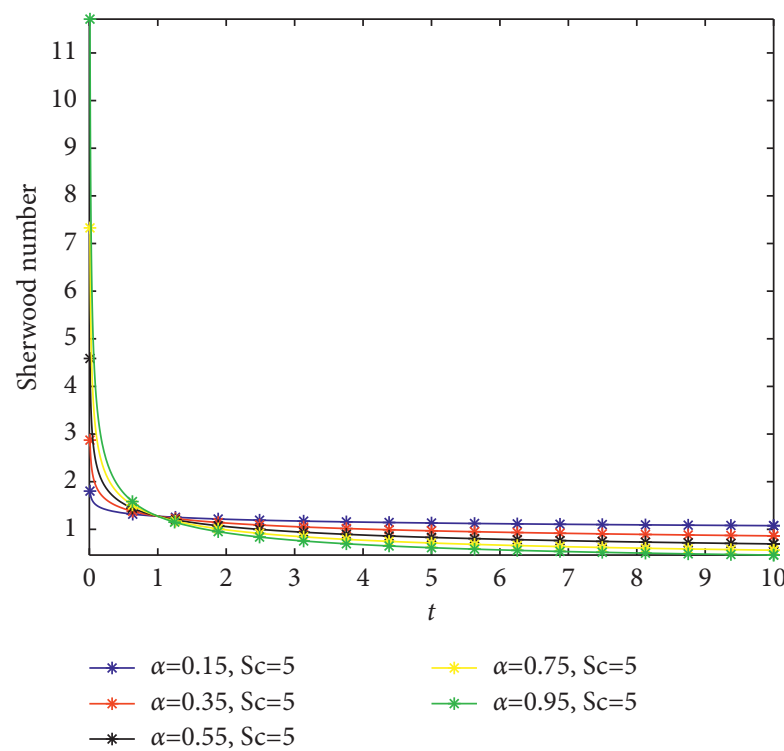

(a)

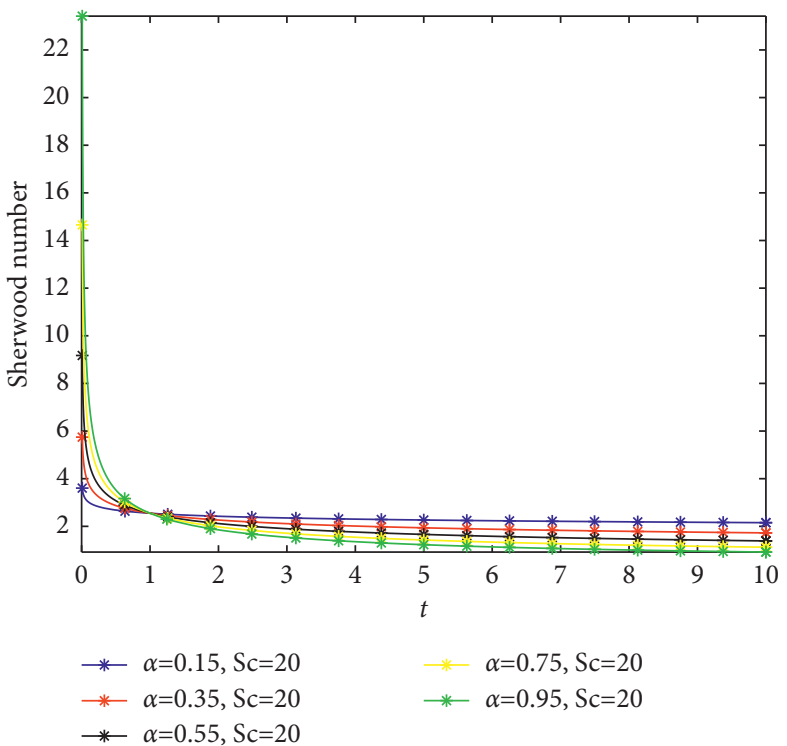

(b)

Figure 17: Sherwood number for different values of order $\alpha$.

\section{Conclusion}

This paper has addressed and focused on the analytical solutions of the fluid model described by the Caputo derivative using the Laplace transform method. The special functions have been used to describe the analytical solutions and their graphical representations. We have analyzed the influence of the order of the Caputo derivative and find that it plays acceleration or retardation effects. These behaviors are due to the accumulation of the memory effect described in the paper. We have also analyzed the influence of the Prandtl and Schmidt numbers in the dynamics of the concentration and the temperature. We find that these numbers generate a decrease in these distributions. We also find that Grashof numbers generate a decrease in the velocities due to the impact of these numbers on the buoyancy forces. In the future direction of research, the analysis adopted in this paper can be addressed with the same model but with different initial and boundary conditions, which play an important role in the form of the exact analytical solutions. The graphical representations have been used in this paper to support all the paper's findings.

\section{Data Availability}

No data were used to support this study.

\section{Conflicts of Interest}

The author declares no conflicts of interest.

\section{References}

[1] X. Wang, Z. Wang, X. Huang, and Y. Li, "Dynamic analysis of a delayed fractional-order SIR model with saturated incidence and treatment functions," International Journal of Bifurcation and Chaos, vol. 28, no. 14, Article ID 1850180, 2018.
[2] S. Qureshi, A. Yusuf, A. A. Shaikh, and M. Inc, "Transmission dynamics of varicella zoster virus modeled by classical and novel fractional operators using real statistical data," Physica A: Statistical Mechanics and Its Applications, vol. 534, Article ID 122149, 2019.

[3] M. A. Imran, S. Sarwar, M. Abdullah, and I. Khan, "An analysis of the semi-analytic solutions of a viscous fluid with old and new definitions of fractional derivatives," Chinese Journal of Physics, vol. 56, no. 5, pp. 1853-1871, 2018.

[4] N. Sene, "Study of a fractional-order chaotic system represented by the Caputo operator," Complexity, vol. 2021, Article ID 5534872, 20 pages, 2021.

[5] K. Saad, D. Baleanu, and A. Atangana, "New fractional derivatives applied to the Korteweg-de Vries and Korteweg-de Vries-Burger's equations," Computational and Applied Mathematics, vol. 37, no. 6, 2019.

[6] N. A. Shah, I. Khan, M. Aleem, and M. A. Imran, "Influence of magnetic field on double convection problem of fractional viscous fluid over an exponentially moving vertical plate: new trends of Caputo time-fractional derivative model," Advances in Mechanical Engineering, vol. 11, no. 7, pp. 1-11, 2019.

[7] I. Khan, N. Ali Shah, and D. Vieru, "Unsteady flow of generalized Casson fluid with fractional derivative due to an infinite plate," The European Physical Journal Plus, vol. 131, no. 6, p. 181, 2016.

[8] K. M. Owolabi and A. Atangana, "On the formulation of Adams-Bashforth scheme with Atangana-Baleanu-Caputo fractional derivative to model chaotic problems," Chaos: An Interdisciplinary Journal of Nonlinear Science, vol. 29, no. 2, Article ID 023111, 2019.

[9] F. Ali, M. Saqib, I. Khan, and N. Ahmad Sheikh, "Application of Caputo-Fabrizio derivatives to MHD free convection flow of generalized Walters'-B fluid model," The European Physical Journal Plus, vol. 131, no. 10, p. 377, 2016.

[10] N. Sene, "Integral balance methods for Stokes' first equation described by the left generalized fractional derivative," Physics, vol. 1, no. 1, pp. 154-166, 2019. 
[11] N. Sene, "Fractional advection-dispersion equation described by the Caputo left generalized fractional derivative," Palestine Journal of Mathematics, vol. 10, no. 2, pp. 562-579, 2021.

[12] N. Sene, "Mathematical views of the fractional Chua's electrical circuit described by the Caputo-Liouville derivative," Revista Mexicana de Física, vol. 67, no. 1, pp. 91-99, 2021.

[13] A. A. Kilbas, H. M. Srivastava, and J. J. Trujillo, Theory and Applications of Fractional Differential Equations, NorthHolland Mathematics Studies, Elsevier, Amsterdam, The Netherlands, 2006.

[14] I. Podlubny, Fractional Differential Equations, Mathematics in Science and Engineering, Academic Press, New York, NY, USA, 1999.

[15] J. Fahd, T. Abdeljawad, and D. Baleanu, "On the generalized fractional derivatives and their Caputo modification," The Journal of Nonlinear Science and Applications, vol. 10, pp. 2607-2619, 2017.

[16] A. Atangana and S. I. Araz, "Extension of Atangana-Seda numerical method to partial differential equations with integer and non-integer order," Alexandria Engineering Journal, vol. 13, 2020.

[17] A. Atangana and D. Baleanu, "New fractional derivatives with nonlocal and non-singular kernel: theory and application to heat transfer model," Thermal Science, vol. 20, no. 2, pp. 763-769, 2016.

[18] M. Caputo and M. Fabrizio, "A new definition of fractional derivative without singular kernel," Progress in Fractional Differentiation and Applications, vol. 1, no. 2, pp. 1-15, 2015.

[19] A. khan, K. Ali Abro, A. Tassaddiq, and I. Khan, "Atanganabaleanu and Caputo fabrizio analysis of fractional derivatives for heat and mass transfer of second grade fluids over a vertical plate: a comparative study," Entropy, vol. 19, no. 8, p. 279, 2017.

[20] S. A. Samiulhaq, S. Ahmad, D. Vieru, I. Khan, and S. Shafie, "Unsteady magnetohydrodynamic free convection flow of a second grade fluid in a porous medium with ramped wall temperature," PLoS One, vol. 9, no. 5, Article ID e88766, 2014.

[21] N. A. Shah, I. Khan, M. Aleem, and M. A. Imran, "Influence of magnetic field on double convection problem of fractional viscous fluid over an exponentially moving vertical plate: new trends of Caputo time-fractional derivative model," Advances in Mechanical Engineering, vol. 11, no. 7, pp. 1-11, 2019.

[22] A. A. Zafar, M. B. Riaz, and M. I. Asjad, "Unsteady rotational flow of fractional Maxwell fluid in a cylinder subject to shear stress on the boundary," Punjab University Journal of Mathematics, vol. 52, no. 2, pp. 21-32, 2018.

[23] A. Hussanan, M. Z. Salleh, I. Khan, R. M. Tahar, and Z. Ismail, "Soret effects on unsteady magnetohydrodynamic mixedconvection heat-and-mass-transfer flow in a porous medium with Newtonian heating," Maejo International Journal of Science and Technology, vol. 9, no. 2, pp. 224-245, 2015.

[24] R. Ridhwan Reyaz, Y. J. Yeou Jiann Lim, A. Q. Ahmad Qushairi Mohamad, M. Muhammad Saqib, and S. Sharidan Shafie, "Caputo fractional MHD Casson fluid flow over an oscillating plate with thermal radiation," Journal of Advanced Research in Fluid Mechanics and Thermal Sciences, vol. 85, no. 2, pp. 145-158, 2021.

[25] M. Narahari and B. K. Dutta, "Effects of thermal radiation and mass diffusion on free convection flow near a vertical plate with Newtonian heating," Chemical Engineering Communications, vol. 199, no. 5, pp. 628-643, 2012.

[26] N. Sene, "Mathematical views of the fractional Chua's electrical circuit described by the Caputo-Liouville derivative," Revista Mexicana de Física, vol. 67, no. 1, pp. 91-99, 2021.
[27] K. A. Abro, "A fractional and analytic investigation of thermodiffusion process on free convection flow: an application to surface modification technology," European Physical Journal Plus, vol. 135, no. 1, pp. 31-45, 2019.

[28] F. Shen, W. Tan, Y. Zhao, and T. Masuoka, "The RayleighStokes problem for a heated generalized second grade fluid with fractional derivative model," Nonlinear Analysis: Real World Applications, vol. 7, no. 5, pp. 1072-1080, 2006.

[29] D. Vieru, C. Fetecau, and C. Fetecau, "Time-fractional free convection flow near a vertical plate with Newtonian heating and mass diffusion," Thermal Science, vol. 19, no. 1, pp. 85-98, 2015.

[30] A. Khalid, I. Khan, A. Khan, and S. Shafie, "Unsteady MHD free convection flow of Casson fluid past over an oscillating vertical plate embedded in a porous medium," Engineering Science and Technology, an International Journal, vol. 18, no. 3, pp. 309-317, 2015.

[31] M. B. Riaz, M. A. Imran, and K. Shabbir, "Analytic solutions of Oldroyd-B fluid with fractional derivatives in a circular duct that applies a constant couple," Alexandria Engineering Journal, vol. 55, no. 4, pp. 3267-3275, 2016.

[32] M. A. Imran, N. A. Shah, I. Khan, and M. Aleem, "Applications of non-integer Caputo time fractional derivatives to natural convection flow subject to arbitrary velocity and Newtonian heating," Neural Computing \& Applications, vol. 30, no. 5, pp. 1589-1599, 2018.

[33] M. A. Imran, S. Sarwar, and M. Imran, "Effects of slip on free convection flow of Casson fluid over an oscillating vertical plate," Boundary Value Problems, vol. 2016, no. 1, p. 30, 2016. 